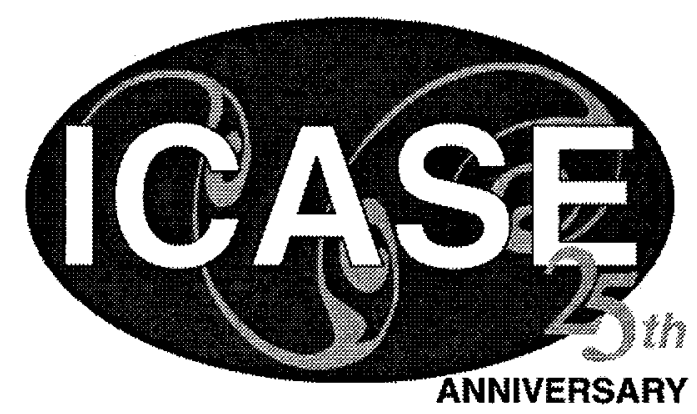

\title{
ON THE ASYMPTOTIC REGIMES AND THE STRONGLY STRATIFIED LIMIT OF ROTATING BOUSSINESQ EQUATIONS
}

\section{A. Babin}
A. Mahalov
B. Nicolaenko
Y. Zhou

NASA Contract No. NAS1-19480

March 1997

Institute for Computer Applications in Science and Engineering NASA Langley Research Center

Hampton, VA 23681-0001

Operated by Universities Space Research Association

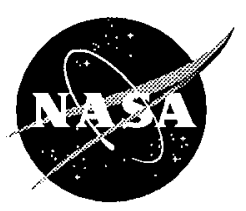

National Aeronautics and Space Administration

Langley Research Center Hampton, Virginia 23681-0001 



\title{
On the Asymptotic Regimes and the Strongly Stratified Limit of Rotating Boussinesq Equations
}

\author{
A. Babin ${ }^{1}$, A. Mahalov ${ }^{1}$, B. Nicolaenko ${ }^{1}$ and Y. Zhou ${ }^{2 *}$ \\ 1. Department of Mathematics, Arizona State University, Tempe, AZ 85287 \\ 2 Institute for Computer Applications in Science and Engineering \\ NASA Langley Research Center, Hampton, VA 23681
}

\begin{abstract}
Asymptotic regimes of geophysical dynamics are described for different Burger number limits. Rotating Boussinesq equations are analyzed in the asymptotic limit of strong stratification in the Burger number of order one situation as well as in the asymptotic regime of strong stratification and weak rotation. It is shown that in both regimes horizontally averaged buoyancy variable is an adiabatic invariant for the full Boussinesq system. Spectral phase shift corrections to the buoyancy time scale associated with vertical shearing of this invariant are deduced. Statistical dephasing effects induced by turbulent processes on inertial-gravity waves are evidenced. The 'split' of the energy transfer of the vortical and the wave components is established in the Craya-Herring cyclic basis. As the Burger number increases from zero to infinity, we demonstrate gradual unfreezing of energy cascades for ageostrophic dynamics. The energy spectrum and the anisotropic spectral eddy viscosity are deduced with an explicit dependence on the anisotropic rotation/stratification time scale which depends on the vertical aspect ratio parameter. Intermediate asymptotic regime corresponding to strong stratification and weak rotation is analyzed where the effects of weak rotation are accounted for by an asymptotic expansion with full control (saturation) of vertical shearing. The regularizing effect of weak rotation differs from regularizations based on vertical viscosity. Two scalar prognostic equations for ageostrophic components (divergent velocity potential and geostrophic departure) are obtained.
\end{abstract}

\footnotetext{
*'This research was supported by the National Aeronautics and Space Administration under NASA Contract No. NAS119480 while the author was in residence at the Institute for Computer Applications in Science and Engineering (ICASE), NASA Langley Research Center, Hampton, VA 23681-0001
} 


\section{Introduction}

The turbulent flows that are subject to rotation and stratification have many important applications in geophysics and engineering (Fernando and Hunt, 1996; Hopfinger, 1989). An important class of geophysical flows can be characterized as strongly rotating and strongly stratified with both effects playing an important role in the dynamics. This is the so called Burger number of order one regimes where the effects of rotation and stratification enter at the same order in asymptotics (McWilliams 1985). One of the major difficulties encountered in understanding dynamics of geophysical flows is the influence of the oscillations generated by the rotation and stratification. One major effect of rotation and stratification is through "phase scrambling" for the wave phase. In this paper rotating Boussinesq equations are analyzed in the asymptotic limit of strong stratification in the Burger number of order one situation as well as in the asymptotic regime of strong stratification and weak rotation.

The very useful and thought provoking multi-scale analyses of rotating/stratified turbulence is presented in Riley et al. (1981), Lilly (1983), McWilliams (1985). In particular, they argue that the velocity field of a rotating, stably stratified fluid may be regarded as a superposition of waves which are modulated on the longer turbulence time scale. In our approach, the collective contribution to the dynamics made by waves is accounted for by rigorous KAM (Kolmogorov-Arnold-Moser) type theory and rigorous estimates of wave resonances and quasi-resonances via small divisors analysis. Our theory handles rigorously 3 -waves resonances, but goes much deeper into the structure of quasi 3-wave resonances and their contributions. This mathematical approach was initiated in Babin, Mahalov and Nicolaenko (henceforth BMN) (1995), Mahalov and Marcus (1995) in the context of geophysical flows. In Bartello (1995), the relative physical importance of different resonances is discussed in depth. In this paper we present the physical predictions and implications of our rigorous mathematical analysis. Interactions between internal waves and the vortical (quasi-geostrophic) modes remain as one of the important questions to be addressed by strong interaction theory (Müller, Holloway et al., 1986; Warn, 1986; Farge and Sadourny, 1989; Lelong and Riley, 1991).

The governing flow equations for rotating stably stratified fluids under the Boussinesq approximation are

$$
\begin{gathered}
\partial_{t} \mathbf{U}+\mathbf{U} \cdot \nabla \mathbf{U}+2 \Omega_{0} e_{3} \times \mathbf{U}=-\nabla p+\rho_{1} e_{3}, \nabla \cdot \mathbf{U}=0, \\
\partial_{t} \rho_{1}+\mathbf{U} \cdot \nabla \rho_{1}=-N_{0}^{2} U^{3},
\end{gathered}
$$

where rotation and mean stratification gradient are aligned parallel to $e_{3}=[0,0,1]$. Here $\mathbf{U}=\left(U^{1}, U^{2}, U^{3}\right)$ is the velocity field and $\rho_{1}$ is the buoyancy variable; $N_{0}$ is the Brunt-Väisälä frequency for constant stratification and $\Omega_{0}$ is the frequency of background rotation, $f_{0}=2 \Omega_{0}$. We focus on inviscid Eqs. (1.1)-(1.2) or with small uniform viscosities. 
Now we introduce useful non-dimensional parameters. Let $U_{h}$ be a characteristic horizontal velocity scale. Let $H$ and $L$ be vertical and horizontal length scales and $a_{3}=H / L$ is the aspect ratio parameter. We define Froude numbers based on horizontal and vertical scales:

$$
F_{h}=U_{h} / L N_{0} \equiv 1 / N, F_{v}=U_{h} / H N_{0}=F_{h} / a_{3}
$$

The classical Rossby and anisotropic Rossby number are defined as follows

$$
R o=U_{h} / 2 L \Omega_{0} \equiv 1 / 2 \Omega \equiv 1 / f, R o_{a}=a_{3} R o .
$$

The time is dimensionalized using the turbulence time scale $L / U_{h}$. In the Burger $O(1)$ regime $R o_{a} \sim F_{h}$. We are not taking $a_{3} \rightarrow 0$; rather its value is fixed by shallowness of the atmosphere, $a_{3} \sim 5 \times 10^{-3}$ to $10^{-2}$ for synoptic scales. Its smallness effectively downsizes $R o_{a} \sim 5 \times 10^{-4}$ to $10^{-3}$, as $R o \sim 0.1$ in midlatitudes. For mesoscales, $L \sim 100 \mathrm{~km}, a_{3} \sim 10^{-1}$ and $R o_{a} \sim 10^{-2}$. The anisotropic Rossby number $R o_{a}$ or/and the vertical Froude number $F_{v}$ control our uniform error estimates.

The Burger number characterizes relative importance of the effects of rotation and stratification (McWilliams, 1985):

$$
B u=R o_{a}^{2} / F_{h}^{2} \equiv R o^{2} / F_{v}^{2} \equiv N^{2} a_{3}^{2} / f^{2}=N_{0}^{2} a_{3}^{2} / f_{0}^{2} .
$$

In Eqs. (1.3)-(1.4) $f=R o^{-1}$ and $N=F_{h}^{-1}$ are dimensionless rotation and Brunt-Väisälä parameters, respectively. The relative importance of rotation/stratification is measured by the Burger number with $B u<<1$ corresponding to rotation dominated and $B u>>1$ corresponding to stratification dominated flows. Herring and Métais (1989) observed horizontal layering of the velocity field in numerical simulations of stratified turbulence, while Bartello et al. (1994) noted the formation of quasi-two-dimensional structures in rotating turbulence.

Fourier series will be used in this paper to represent physical fields in a parallepiped $[0,2 \pi] \times\left[0,2 \pi / a_{2}\right] \times$ $\left[0,2 \pi / a_{3}\right], 0<a_{2} \leq 1$. We denote $k_{2} / a_{2}$ by $\breve{k}_{2}, k_{3} / a_{3}$ by $\breve{k}_{3}$. Following Bartello (1995), it is useful to distinguish between three sets of wavevectors $\breve{k}=\left(k_{1}, k_{2} / a_{2}, k_{3} / a_{3}\right),|\breve{k}|^{2}=k_{1}^{2}+k_{2}^{2} / a_{2}^{2}+k_{3}^{2} / a_{3}^{2}$ : the barotropic set $\left\{k: k_{3}=0\right\}$, the set with only vertical variability $\left\{k: k_{1}=k_{2}=0\right\}$, and the remaining baroclinic vectors $\left\{k: k_{1}^{2}+k_{2}^{2} \neq 0, k_{3} \neq 0\right\}$. Then the operation of vertical averaging corresponds to projection on barotropic fields; the operation of horizontal averaging corresponds to projection on fields with only vertical variability. In this paper as well as in our previous work $(B M N, 1996 a, b, d)$ we emphasize the important role of operations of vertical and horizontal averaging in investigations of rotating and stratified flows.

Regimes of geophysical dynamics presenting the global picture for small Froude or Rossby numbers are shown in Figure 1 which summarizes the physical implications of our mathematical analysis. Since we are 


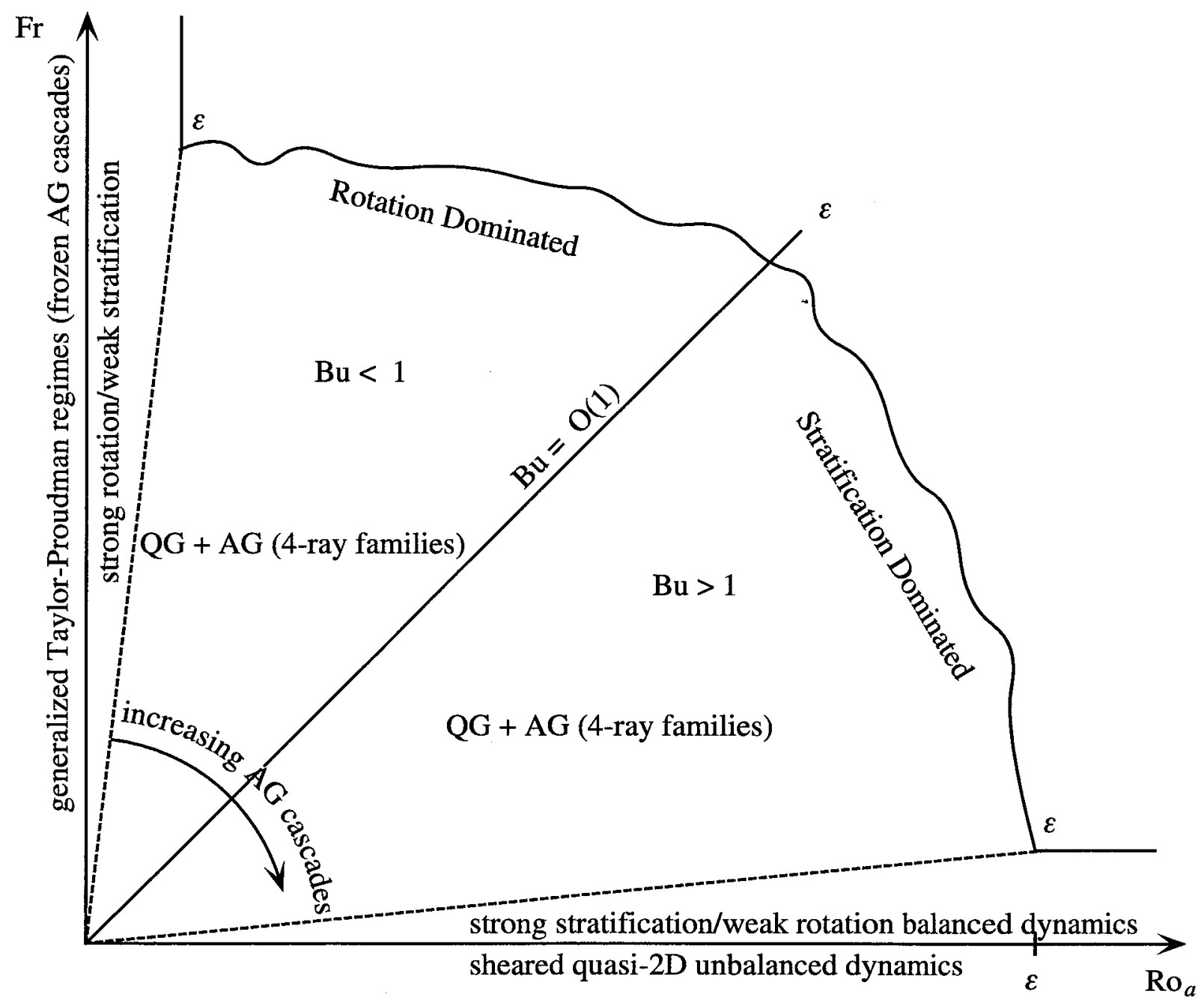

Fig. 1. Geophysical Dynamics: the global picture for small Froude or small Rossby regimes. 
not taking $a_{3} \rightarrow 0$, either $F_{h}$ or $F_{v}$ can be used in description of asymptotic regimes. Then $F r$ denotes either of these numbers. The rotation dominated case corresponding to $R o_{a} \rightarrow 0, B u \rightarrow 0$ and $\mathrm{Fr}$ finite was considered in BMN (1995), Zhou (1995), BMN (1996a, c, d, e), Mahalov and Zhou (1996) (Figure 1 , vertical axis). In this case we proved the generalized Taylor-Proudman theorem establishing splitting between vertical averages of $\mathbf{U}, \rho_{1}$ (two dimensional- four component, 2D-4C barotropic fields) and reduced ageostrophic field. Following Reynolds and Kassinos (1996) 2D-4C refers to fields with four components depending on two variables $x_{1}$ and $x_{2} ; 3 \mathrm{D}-2 \mathrm{C}$ refers to fields with two components depending on three variables $x_{1}, x_{2}$ and $x_{3}$ etc. By splitting we mean that the barotropic field decouples from the ageostrophic one, without feedback from the latter onto the former, to the lowest order. In the limit $N \rightarrow 0$, the usual quasi-geostrophic field does reduce to vertically averaged fields; this is what is meant by "geostrophic" in this limiting context. In this limit vertical shearing is fully controlled which is reflected in adiabatic invariants associated with vertical shearing (exact conservation laws in reduced equations), with $\mathbf{U}^{\dagger}=\left(\mathbf{U}, \rho_{1}\right)$

$$
\frac{d}{d t} \int\left|\frac{\partial \mathbf{U}^{\dagger}}{\partial x_{3}}\right|^{2} d x_{1} d x_{2} d x_{3}=0
$$

In the limit $N \rightarrow 0$ the temperature decouples from the dynamics and behaves as a passive scalar. The dynamics of vertically averaged velocity fields reduce to classical 2D-3C Euler equations and are subject to inverse energy cascades in $U^{1}, U^{2}$ as in 2D turbulence. For $B u \rightarrow 0$ we have shown rigorously that energy cascades for the ageostrophic (AG) field are completely frozen in $x_{3}$ and the dynamics is pure phase turbulence (BMN, 1996a,c,d,e); freezing of energy cascades in $x_{3}$ for the "baroclinic" component follows from (1.6). In pure phase turbulence, the amplitudes of the ageostrophic modes remain constant in absolute values; turbulent dynamics are restricted to the phases of the ageostrophic modes. The ageostrophic field is phase locked to phases associated with vertically averaged vertical vorticity and vertical velocity which are advected by $2 \mathrm{D}$ turbulence of vertically averaged fields. There is no slaving of the amplitudes of ageostrophic modes by the $2 \mathrm{D}$ turbulence, only phase locking. We calculated Doppler phase shifts induced by turbulence of vortical (vertically averaged) fields on inertio-gravity waves in this limit. In the case of 3D rotating Euler in the small anisotropic Rossby number situation we described regimes with no energy flux in the ageostrophic (AG) component and formation of KAM-type regimes with frozen in $x_{3}$ ageostrophic cascades (pure phase turbulence, frozen turbulence). Similar freezing of energy cascades was observed by Farge (1988) in the context of rotating shallow-water equations and in Pushkarev and Zakharov (1996) in numerical experiments describing turbulence of capillary waves.

Next is the regime of strong rotation and weak stratification as shown in Figure 1. Besides the operation of vertical averaging there is a piece of 3DQG (quasi-geostrophic, Pedlosky 1987) which plays an important role in the dynamics. It is formally obtained by expanding $3 \mathrm{DQG}$ equations in a small parameter $N / f$. 
This procedure is similar to the one described in Section 4 for the regime of strong stratification and weak rotation. The corresponding reduced equations, higher order corrections and mathematical convergence results for the case of balanced and unbalanced initial data are presented in Avrin, Babin, Mahalov and Nicolaenko (henceforth ABMN) (1996).

As the effects of stratification are increased further (see Fig. 1) AG cascades become possible. In the limit of strong rotation and strong stratification corresponding to $R o_{a} \rightarrow 0, F r \rightarrow 0$, but $B u=O(1)$ we established splitting between 3DQG and the reduced ageostrophic field (BMN, 1996b) confirming the similar splitting for rotating shallow-water equations which we obtained in Mahalov and Marcus (1995) and for rotation-dominated case in BMN (1995), (1996a). Again, by splitting we mean that the QG field decouples from the ageostrophic one. Dynamics of AG further splits along uncoupled resonant quadruplets of Fourier rays with AG energy conserved on each resonant quadruplet of rays. Energy cascades are now allowed (unfrozen) for the ageostrophic field but they are restricted to families of 4 rays in Fourier space. Energy transfers (direct and inverse cascades) are not prohibited but restricted on uncoupled families of resonant four rays for AG field. Direct energy cascades of AG field provide mechanism for nonlinear geostrophic adjustment. This is fundamentally different from the rotation dominated regimes where AG cascades are frozen. Spectral differential molecular viscosities for $Q G$ and AG fields with explicit dependence on the rotation/stratification parameters behave differently. Let $\nu_{1}$ and $\nu_{2}$ be the kinematic viscosity and the heat conductivity, respectively; the ratio $\nu_{1} / \nu_{2}$ is known as the Prandtl number. Through a simple computation of 2-wave resonances in the Craya-Herring basis (Lesieur, 1987) the effective differential spectral molecular viscosities $\nu_{Q G}$ and $\nu_{A G}$ are given by

$$
\nu_{Q G}(n)=\nu_{2}+\left(\nu_{1}-\nu_{2}\right) \frac{\left|\check{n}^{\prime}\right|^{2}}{\left|\check{n}^{\prime}\right|^{2}+\mu^{2} \check{n}_{3}^{2}}, \nu_{A G}(n)=\nu_{1}+\left(\nu_{2}-\nu_{1}\right) \frac{\left|\check{n}^{\prime}\right|^{2}}{\left|\check{n}^{\prime}\right|^{2}+\mu^{2} \check{n}_{3}^{2}}
$$

where $\mu=f / N,\left|\check{n}^{\prime}\right|^{2}=n_{1}^{2}+n_{2}^{2} / a_{2}^{2}$. It shows that dissipation affects QG and AG fields differently. This impacts on direct numerical simulations of $Q G$ fields in the context of numerical simulations of atmospheric flows.

Partial control of vertical shearing is obtained allowing us to prove long time existence of solutions of inviscid Boussinesq equations (BMN, 1996b and Section 6). Also, a flow which is initially wave dominated remains wave dominated even through decay (confirming Métais et al., 1996). In Section 3 we show that horizontally averaged buoyancy variable $\bar{\rho}_{1}$ is an adiabatic invariant (this result is true for all resonances including 3 wave resonances); we calculate Doppler phase corrections associated with $\frac{d}{d x_{3}} \bar{\rho}_{1}$ to a linear profile (constant $N_{0}$ ). This confirms and generalizes the work of Legras (1980) on phase shifts who showed the existence of statistical dephasing effects induced by turbulent processes on Rossby waves. Frequency shifts induced by turbulent processes on inertial waves were calculated in BMN (1996a); the case of frequency shifts induced on waves in rotating shallow-water equations was considered in BMN (1996c, e). As the 
effects of stratification are increased (see Fig. 1) vertical shearing dynamics in the ageostrophic field increase and is conveniently characterized using the divergent velocity potential $\chi\left(t, x_{1}, x_{2}, x_{3}\right)$ given by the formula $\chi=\left(-\Delta_{h}\right)^{-1} \partial U^{3} / \partial x_{3}$ which is coupled to geostrophic departure. The geostrophic departure $-\nabla_{h}^{2} \rho_{1}+$ $f \frac{\partial}{\partial x_{3}} \operatorname{curl} \mathbf{U} \cdot e_{3}$ characterizes imbalance in the vertical motion or omega equation (Eqs. (1.16)-(1.17)). Up to a normalization, the divergent velocity potential $\chi$, the geostrophic departure and 3DQG mode form the Craya-Herring cyclic basis which is used in this paper to represent physical fields. This is further described below.

In the inviscid regime $F r \rightarrow 0, R o_{a}=O(1), B u \rightarrow+\infty$ (Figure 1, horizontal axis) we prove that there is no bound on vertical shearing associated with the dynamics of 3D-2C (3 dimensional, 2 components) decoupled pancakes (parametrized in $x_{3}$ ) with different pressures at every level; this leads to unbalanced dynamics at the lowest order. There is no saturation of the exponential build-up of vertical enstrophy (in small vertical scales) for $\mathrm{AG}$ dynamics as the latter is coupled to the quasi $2 \mathrm{D}$ field thru $\partial U_{Q 2 D}^{1} / \partial x_{3}, \partial U_{Q 2 D}^{2} / \partial x_{3}$. The major problem is lack of boundedness of vertical shearing in quasi 2D equations (Lilly, 1983). We show that horizontally averaged $\bar{U}^{1}\left(x_{3}\right), \bar{U}^{2}\left(x_{3}\right), \bar{\rho}_{1}\left(x_{3}\right)$ are adiabatic invariants providing a feedback onto AG turbulence. $\bar{U}^{1}, \bar{U}^{2}$ are adiabatic invariants only if $\Omega=0$; otherwise, they undergo rigid $\Omega$-rotation (this result holds for all resonances including the 3 wave resonances). However, these adiabatic invariants are not enough to saturate vertical shearing. Worse the lack of boundedness of $\partial U_{Q 2 D}^{1} / \partial x_{3}, \partial U_{Q 2 D}^{2} / \partial x_{3}$ leads to explosive exponential growth of the AG dynamics. Of course, control of vertical shearing can be achieved trivially by introducing vertical viscosity; however, this corresponds to a non-physical laboratory set-up rather than the real atmosphere (A. Majda, private communication), or a poorly-resolved (in $x_{3^{-}}$scales) numerical model (P. Bartello, private communication).

In the intermediate asymptotic regime corresponding to strong stratification and weak rotation $(B u \rightarrow$ $+\infty, f / N$ small) the effects of weak rotation on the dynamics are accounted for by an asymptotic expansion in a small parameter $\mu=f / N$ (Section 4). Full saturation of vertical shearing is obtained for all times. Equations describing balanced dynamics are intermediate between $3 \mathrm{DQG}$ equations valid in the regime $B u=$ $O(1)$ and quasi-2D decoupled pancakes without any control of vertical shearing (Lilly, 1983). In this paper we show how weak rotation regularizes vertical shearing and calculate its effects on AG dynamics. Our reduced equations have a conservation law associated with vertical shearing which allows to control AG vertical scales for all times. There is no need to resort to vertical viscosity as the principal stabilizing mechanism (Reynolds number $R e \sim 10^{12}$ in atmospheric flows). Two scalar decoupled equations for ageostrophic components (divergence velocity potential and geostrophic departure) are obtained. These equations have coefficients depending on regularized quasi-2D fields and can be used for more accurate and robust numerical simulations of geophysical flows in the regime of strong stratification and weak rotation. AG dynamics is 
driven by regularized vertical shearing of the pancakes.

In this paper we emphasize physical predictions which follow from rigorous mathematical analysis of Boussinesq equations in the strongly rotating/stratified $B u=O(1)$ regime as well as in the asymptotic regime of strong stratification and weak rotation. The mathematical theory is based on rigorous small divisor estimates and KAM type (Kolmogorov-Arnold-Moser) theoretical considerations to rigorously control wave resonances, especially the 3 -waves of the fast-fast-fast resonances (Figure 2).

On the physical side, for the $B u=O(1)$ regimes discussed in Sections 3 and 5, we establish statistical dephasing effects induced by turbulent processes on inertio-gravity waves with 3DQG turbulence acting to renormalize both frequency and viscosity of the waves. We generalize the work of Legras (1980), Carnevale and Martin (1982). In particular, we calculate Doppler phase corrections associated with the fact that horizontally averaged buoyancy variable $\bar{\rho}_{1}$ is an adiabatic invariant. Namely, $\bar{\rho}_{1}\left(x_{3}\right)$ has a $O(\epsilon)$ variation on large times when $R o_{a} \sim F_{h} \sim \epsilon$. Rigorous mathematical analysis based on small divisor estimates shows that 3 waves fast-fast-fast resonances are rare in the $B u=O(1)$ atmospheric regimes (BMN, 1996b and Section 6) as well as in $B u<<1$ regimes (BMN, 1996a). In fact, just switching on even weak rotation destroys the 3 -waves resonances found in the pure stratified case $f=0$. One of the hardest parts of our analysis is to estimate the total probabilities of quasi-resonances, that is the width of Arnold tongues coming out of points (set of measure zero) where 3 wave resonances are possible (see Figure 2). These resonances are not neglected but rather weights are assigned to them according to their importance (BMN, 1996d and Section 6). Even 3 waves resonances do not alter the global picture: they correspond to higher order corrections ("Arnold drift").

The width of Arnold tongues scales algebraically with $R o_{a}$ (see Corollary 6.1 and Figure 2). For synoptic scales at midlatitudes, $L \approx 2000 \mathrm{~km}, H \approx 10 \mathrm{~km}$, the width (normalized probability of a 3 wave resonance) is of order $(H / L)^{2}=\left(5 \times 10^{-3}\right)^{2}$ and it is of order $\left(10^{-2}\right)^{2}$ at mesoscales $(L \approx 100 \mathrm{~km}) ; 3$ wave interactions become significant only for tall columns (see Section 6). In regions free of fast-fast-fast interactions (set of full Lebesgue measure) nonlinear geostrophic adjustment takes place via "catalytic" interactions between one QG mode and two AG modes confirming the insight and numerical simulations of Bartello (1995). Bartello (1995) also discusses fast-slow-slow interactions, which are non-resonant. In our work, they appear at the next order in $R o_{a}$ or $1 / N$ at $B u=O(1)$, and contribute to the feedback of the ageostrophic field onto the QG one (ABMN, 1996). Inside Arnold tongues where fast-fast-fast 3 wave interactions are possible we expect Arnold drifts associated with neglected higher order resonances. Guided by KAM theory in finite dimensional systems and the fact that Arnold tongues are very narrow in our problem (Section 6), we expect that these drifts will be slowly evolving (cf. Figure 2). Thus we expect the dynamical picture to be intact even inside Arnold tongues where 3 wave interactions are possible. Our analysis (Section 6 ) proves that 3 wave 


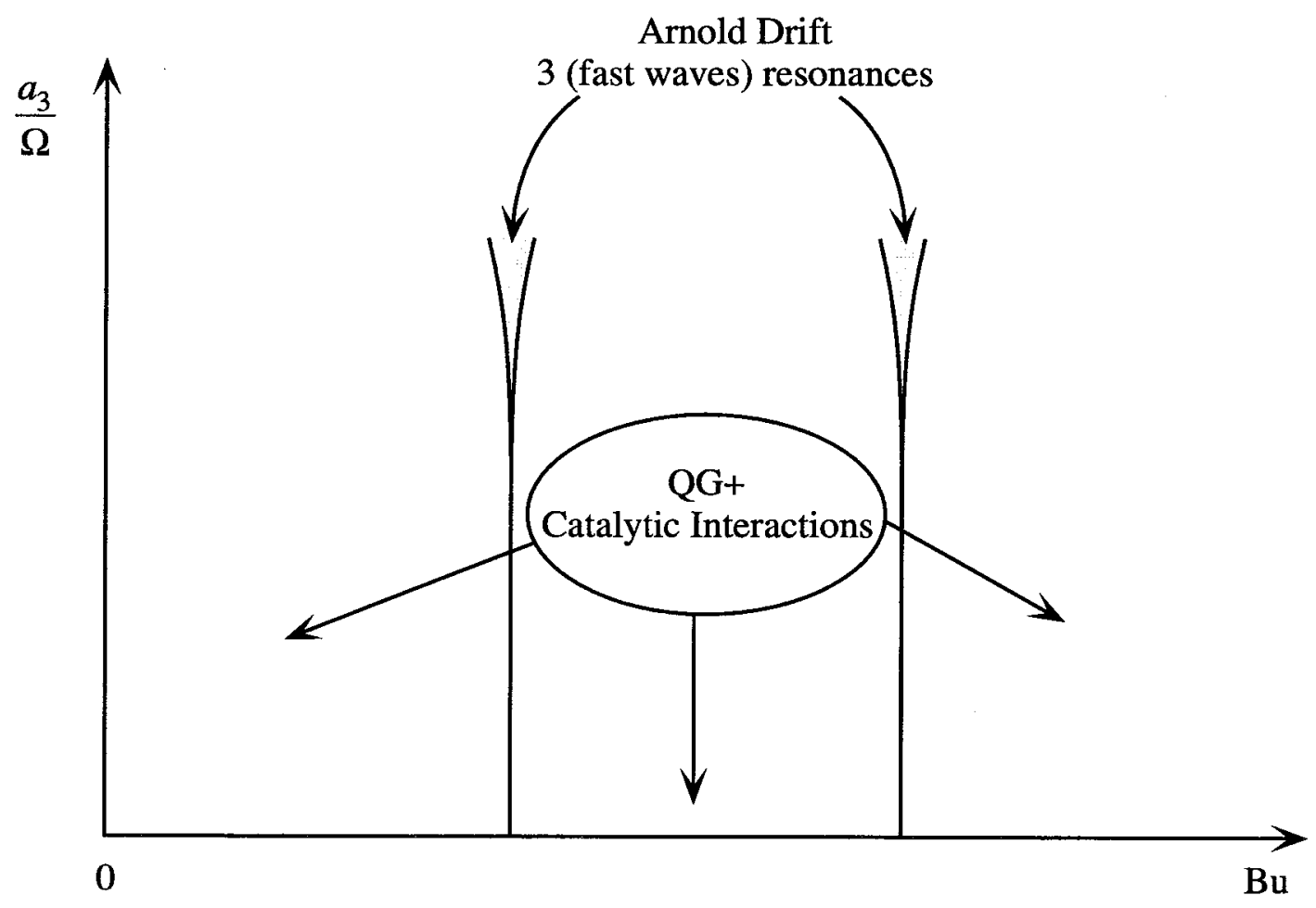

Fig. 2. The global picture of resonances for bounded Burger numbers. 
interactions will not contaminate large scale dynamics. Breakdowns can occur only locally corresponding to small horizontal scales. In this case a local anisotropic Rossby number based on local horizontal scale will become large and both local breakdown and collapse leading to fully 3D dynamics (locally) will be possible. We have analyzed such 3D instabilities in an idealized case of rotating columnar flows (Mahalov, 1993). Here our rigorous mathematical analysis amounts to the following: the probability of such a localized breakdown to $3 \mathrm{D}$ turbulence to extend to larger scales via a catastrophic inverse cascade is very small. This is indeed what is observed in the atmosphere, where near balanced dynamics are sustained for all times in the larger scales. The regime $F r \rightarrow 0, R o_{a}=O(1), B u \rightarrow \infty$ requires a special attention. In this regime 3DQG equations degenerate to quasi-2D equations lacking control of vertical shearing and leading to unbalanced dynamics at the lowest order. To this end, a regularization based on weak rotation is proposed in Section 4 .

In BMN (1996b) we established splitting between 3DQG and reduced ageostrophic field; here we show that the structure of reduced ageostrophic equations (via Craya-Herring cyclic basis) implies upscale (inverse) energy transfer of rotational (vortical) energy via QG mode versus downscale cascade of wave energy via slow-fast-fast resonant catalytic interactions (following Bartello, 1995 notations). In cases $B u=O(1)$ and $B u>>1$, AG field satisfies uncoupled families of equations on 4 rays in Fourier space. As the effects of stratification are increased direct cascade of AG energy (along these rays) toward small scales is enhanced. In Section 5 we introduce the anisotropic phase coherence tensor and model anisotropy in $B u=O(1)$ regimes of geophysical turbulence. There is a spectral gap (i.e. a power law scaling break) between QG and AG spectrum with AG spectrum being shallower than the typical $k^{-3}$ QG spectrum; it varies smoothly between $k^{-2}$ and $k^{-5 / 3}$ which is in agreement with numerical simulations of Métais et al. (1996), Bartello et al. (1996).

A possible emerging picture of Burger $O(1)$ turbulence is that 3DQG turbulence being a guiding center is corrupted by phase turbulence and Doppler phase shifts; with the dynamics of the AG field being constrained to a uncoupled 4-rays families, with direct AG cascade restricted to the latter. The feedback of AG field on QG dynamics can be computed at next order in $F r$ or $R o_{a}(\mathrm{ABMN}, 1996)$.

On the mathematical side, the challenge is to prove that dynamics of limit equations uniformly closely approximate geophysical turbulence in regimes:

1. $N=O(1), \Omega>1$ (rotation dominated regime), $F r=1 / N$.

2. $N>>1, \Omega>>1, B u=O(1)$ (strongly rotating/stratified Burger number of order one regime).

One needs uniform error estimates in Froude and Rossby numbers. It is important to realize that "weak" convergence results, "filtered" and o(1) error estimates cannot achieve this. In BMN (1996 a,b,d) and here (Section 6) we obtain strong convergence results with uniform error estimates on sets of full Lebesgue measure with initial data being in the Sobolev space $H^{\tau}$ (and even $H^{4}$ ) for both $B u=O(1)$ and rotation dominated 
regimes. This is in contrast with the work of Embid and Majda (1996) where only local existence results are established. Following general theorems of Schochet (1994), they state a pointwise convergence theorem with $o(1)$ error on a time interval $[0, T]$; since they include (for $B u=O(1)$ ) 3-wave resonances they cannot give any uniform error estimates in the parameters. Our theory handles rigorously 3 -waves resonances, but goes much deeper into the structure of quasi 3-wave resonances and their contributions. Moreover, the interval $[0, T]$ in Embid and Majda (1996) is small where $T$ can be as bad as any local time of existence of original $3 D$ Euler/Boussinesq equations, and it is in fact limited by potential $3 D$ vortex singularities of the full $3 D$ Euler. Embid and Majda (1996) miss the regularizing effects of strong rotation and stratification. As $R o_{a}$ and $F_{h} \rightarrow 0, B u=O(1)$ we prove that $T \rightarrow+\infty$ for (1.1)-(1.2). We agree with them only as to the QG operator decoupling, which we obtained independently in BMN (1996b) for Boussinesq equations.

Now we describe the structure of reduced equations which will be derived in Section 3 for $B u=O(1)$. In the reduced equations the total velocity splits into the quasi-geostrophic field $\mathbf{w}_{Q G}(t)$ satisfying $3 \mathrm{DQG}$ (quasi-geostrophic) equations

$$
\partial_{t} \mathbf{w}_{Q G}=\mathbf{B}_{1}\left(\mathbf{w}_{Q G}, \mathbf{w}_{Q G}\right)
$$

and the ageostrophic component satisfying in principle general equations of the type:

$$
\partial_{t} \mathbf{w}_{A G}=\mathbf{B}_{2}\left(\mathbf{w}_{Q G}, \mathbf{w}_{A G}\right)+\mathbf{B}_{3}\left(\mathbf{w}_{A G}, \mathbf{w}_{A G}\right)
$$

In the case when the balanced $Q G$ dynamics are absent, $\mathbf{w}_{Q G}(t)=0$, Eqs. (1.9) reduce to equations describing wave turbulence (e.g. Zakharov et al., 1992):

$$
\partial_{t} \mathbf{w}_{A G}=\mathbf{B}_{3}\left(\mathbf{w}_{A G}, \mathbf{w}_{A G}\right) .
$$

We prove that (1.9) holds only within very narrow Arnold tongues (Figure 2) with infinitesimal probability (Section 6); we do not just drop $\mathbf{B}_{3}$ with some ad-hoc scaling argument. In the real atmosphere situation a near balance state is sustained and the quasi-geostrophic (balanced) field is present and it is dominant. In Babin, Mahalov and Nicolaenko (1996b) and here (Section 6) it is shown using small divisor estimates that for almost all $B u$ and $a_{3}=H / L$ only "catalytic" interactions rule AG dynamics:

$$
\partial_{t} \mathbf{w}_{A G}=\mathbf{B}_{2}\left(\mathbf{w}_{Q G}, \mathbf{w}_{A G}\right)
$$

where $\mathbf{w}_{Q G}(t)$ is a solution of $3 \mathrm{DQG}$ equations. Such interactions do not influence the slow QG modes, but act to transfer fast AG energy downscale (Bartello, 1995). Further, for all $B u$, all $a_{3}$ and almost all domain aspect ratios $a_{2}, \mathbf{B}_{2}\left(\mathbf{w}_{Q G}, \mathbf{w}_{A G}\right)$ splits in Fourier space into uncoupled, restricted interaction operators on 4 
rays families

$$
\left(\begin{array}{l}
m_{1} \\
m_{2} \\
m_{3}
\end{array}\right)=\lambda\left(\begin{array}{ccc} 
\pm 1 & 0 & 0 \\
0 & \pm 1 & 0 \\
0 & 0 & \pm 1
\end{array}\right)\left(\begin{array}{l}
n_{1} \\
n_{2} \\
n_{3}
\end{array}\right) .
$$

In Eqs. (1.11) direct cascades of energy are allowed for $\mathbf{w}_{A G}$ through $\mathbf{B}_{2}\left(\mathbf{w}_{Q G}, \mathbf{w}_{A G}\right)$. Wave energy cascades toward smaller scales and is subject to strong dissipation in AG mode, via the anisotropic viscosity $\nu_{A G}$ in (1.7).

An important observation on the nature of interactions in Eqs. (1.11) and their impact on AG dynamics (AG cascades/frozen AG turbulence) is now in order. The nature of interactions in Eqs. (1.11) is fundamentally different in rotation dominated and stratification dominated regimes. In the rotation dominated case (see Fig. 1) corresponding to $R o_{a} \rightarrow 0, B u \rightarrow 0$, and $F r$ finite interactions in Eqs. (1.11) for $n$ th, $n=\left(n_{1}, n_{2}, n_{3}\right)$ Fourier mode are restricted to wavenumbers $\check{m}=\left(m_{1}, \check{m}_{2}, \breve{m}_{3}\right)$ satisfying $m_{3}= \pm n_{3}$, $\left|\check{m}^{\prime}\right|=\left|\check{n}^{\prime}\right|, k+m=n(\mathrm{BMN}, 1996 \mathrm{a})$. Thus, modes $m$ which interact with a given mode $n$ lie on the same energy shell as $n$. Interactions in (1.11) are localized in Fourier space and do not extend to zero or infinity. This special nature of interactions in the rotation dominated regime implies freezing of energy cascades in $x_{3}$ in Eqs. (1.11). Similar freezing of energy cascades of AG field (frozen AG turbulence) was proven for rapidly rotating shallow-water equations in Mahalov and Marcus (1995), BMN (1996c). In particular, it was shown that in the case of rapidly rotating shallow-water equations interactions in $\check{n}^{\prime}=\left(n_{1}, \breve{n}_{2}\right)$ AG equation are restricted to wavenumbers $\breve{m}^{\prime}=\left(m_{1}, \check{m}_{2}\right)$ satisfying $\left|\breve{m}^{\prime}\right|=\left|\check{n}^{\prime}\right|, k^{\prime}+m^{\prime}=n^{\prime}$.

The nature of interactions in (1.11) drastically changes when stratification is increased (see Figure 1). As shown in this paper, in the $B u=O(1)$ as well as $B u>>1$ regimes interactions for the $n$-th mode in (1.11) are restricted to four rays families given by (1.12) for almost all $a_{2}$. Thus, interacting modes lie on families of four rays connecting 0 and infinity in Fourier space. AG energy cascades are now possible along these rays from small wavenumbers (large scales) to large wavenumbers (small scales).

Vertical shearing operations in $\mathbf{B}_{2}\left(\mathbf{w}_{Q G}, \mathbf{w}_{A G}\right)$ are conveniently expressed in terms of divergent velocity potential $\chi$. We recall that $\chi$ and $\psi$ are related to a horizontal velocity field $\mathbf{U}_{h}$ by the formula $\mathbf{U}_{h}=$ $e_{3} \dot{\times} \nabla_{h} \psi+\nabla_{h} \chi$. The operator $\mathbf{B}_{2}\left(\mathbf{w}_{Q G}, \mathbf{w}_{A G}\right)$ takes an especially simple form in the Craya-Herring cyclic basis. In this basis the first component of $\mathbf{w}_{A G}$ is simply the divergent velocity potential $\chi=\left(-\Delta_{h}\right)^{-1} \frac{\partial U_{A G}^{3}}{\partial x_{3}}$ or in Fourier space

$$
\chi_{n}=-i \frac{\check{n}_{3}}{|\check{n}|} \frac{1}{\left|\check{n}^{\prime}\right|} w_{n}^{1}
$$

where $\check{n}^{\prime}=\left(n_{1}, n_{2} / a_{2}\right)$, and the components of the full field in the Craya-Herring basis are $\left(w_{n}^{0}, w_{n}^{1}, w_{n}^{2}\right)$. Here $w_{n}^{0}$ is the component of the quasi-geostrophic mode and $w_{n}^{1}, w_{n}^{2}$ are the ageostrophic components. The 
important role of $\chi$ in our $A G$ operator $\mathbf{B}_{2}$ ties with the more classical theory of balanced models (Gent and McWilliams, 1983a and 1983b) and the findings of Farge and Sadourny (1989), Farge (1988) on effects of inertio-gravity waves and rotation on two-dimensional turbulence.

The second component $w^{2}$ of $\mathbf{w}_{A G}$ in the Craya-Herring basis is related in a simple way to the vertical motion or omega equation. We recall that the vertical velocity does not appear in 3DQG equations and the whole purpose of manipulations leading to the omega equation is to obtain a diagnostic equation from which vertical velocity component can be calculated (e.g. Holton, 1992; Gent and McWilliams, 1983a). We briefly recall these calculations starting from Eqs. (1.1)-(1.2) written in the form $\left(f_{0}=2 \Omega_{0}\right)$

$$
\begin{gathered}
\partial_{t} \mathbf{U}+f_{0} e_{3} \times \mathbf{U}-\rho_{1} e_{3}=-\nabla p-\mathbf{U} \cdot \nabla \mathbf{U}, \nabla \cdot \mathbf{U}=0 \\
\partial_{t} \rho_{1}+N_{0}^{2} U^{3}=-\mathbf{U} \cdot \nabla \rho_{1} .
\end{gathered}
$$

First, we apply the operator $f_{0} \frac{\partial}{\partial x_{3}} \mathrm{curl}$ to Eq. (1.14) and obtain a scalar equation by projecting the result on $e_{3}$. Then we apply horizontal Laplacian to Eq. (1.15). Recalling that $\operatorname{curl}\left(e_{3} \times \mathbf{U}\right) \cdot e_{3}=-\frac{\partial U^{3}}{\partial x_{3}}$, we obtain by subtraction

$$
\partial_{t}\left(-\nabla_{h}^{2} \rho_{1}+f_{0} \frac{\partial}{\partial x_{3}} \omega_{3}\right)-\left(N_{0}^{2} \nabla_{h}^{2}+f_{0}^{2} \frac{\partial^{2}}{\partial x_{3}^{2}}\right) U^{3}=G
$$

where $G$ denote nonlinear terms; $\omega_{3}=\operatorname{curl} \mathbf{U} \cdot e_{3}$ is vertical component of the vorticity vector. The omega equation is obtained by dropping $\partial_{t}\left(-\nabla_{h}^{2} \rho_{1}+f_{0} \frac{\partial}{\partial x_{3}} \omega_{3}\right)$ in Eqs. (1.16). Then we obtain a diagnostic equation which is used to determine vertical velocity $U^{3}$ :

$$
-\left(N_{0}^{2} \nabla_{h}^{2}+f_{0}^{2} \frac{\partial^{2}}{\partial x_{3}^{2}}\right) U_{3}=G
$$

Up to a normalization constant, second mode in the Craya-Herring basis is precisely the geostrophic departure $-\nabla_{h}^{2}$ (buoyancy) $+f_{0} \frac{\partial}{\partial x_{3}}$ (vertical vorticity). It characterizes unbalance in Eqs. (1.16)-(1.17). The geostrophic departure has the form $\left|\check{n}^{\prime}\right|^{2} \rho_{1, n}-f_{0} n_{3}\left(\check{n} \times \mathbf{U}_{n}^{\dagger}\right) \cdot e_{3}=N_{0}\left|\check{n}^{\prime}\right|^{2} \rho_{n}-f_{0} n_{3}\left(\check{n} \times \mathbf{U}_{n}^{\dagger}\right) \cdot e_{3}$ in Fourier space. Since $N_{0}\left|\check{n}^{\prime}\right|^{2} \rho_{n}-f_{0} n_{3}\left(\check{n} \times \mathbf{U}_{n}^{\dagger}\right) \cdot e_{3}=\omega_{n}|\check{n}|\left|\check{n}^{\prime}\right| \mathbf{U}_{n}^{\dagger} \cdot q_{2 n}$, the geostrophic departure up to a normalization is precisely the mode $q_{2}$ in the Craya-Herring cyclic basis (see Eqs. (2.9)).

This paper is organized as follows. In Sec. 2 we recast the Boussinesq equations in the Craya-Herring cyclic basis. This intrinsic coordinate system is particularly convenient for investigations of adiabatic conservation laws and global decoupling. Next in Sec. 3, we develop a methodology and procedure for studying rotating Boussinesq equations in the strongly stratified limit. Here, the dynamical decoupling between the vortical and inertial-gravity wave components of the total field is achieved. It is shown that horizontally averaged buoyancy variable is an adiabatic invariant in the asymptotic state and phase corrections to buoyancy time scale which are associated with vertical shear of the horizontally averaged buoyancy are obtained. 
In Section 4 we analyze an intermediate asymptotic regime of strong stratification and weak rotation with effective saturation of vertical shearing of pancakes by weak rotation (no vertical viscosity needed). The energy spectrum and spectral eddy viscosity models are deduced in Section 5. Finally, in Section 6 we describe the uniform convergence results and the regularization of Euler-Boussinesq equations, as $F_{h} \rightarrow 0$, $R o_{a} \rightarrow 0, B u=O(1)$. These results require much less differentiability than in BMN (1996a): now only four derivatives on initial data are required for the most general convergence results on arbitrary long times.

\section{Boussinesq equations in the Craya-Herring cyclic basis}

We introduce a change of variables $\rho_{1}=N \rho$ (Métais and Herring 1989) and combine velocity and buoyancy variable in one variable $\mathbf{U}^{\dagger}=(\mathbf{U}, \rho)$ after which Eqs. (1.1)-(1.2) written in non-dimensional variables take more symmetric form:

$$
\partial_{t} \mathbf{U}^{\dagger}+\mathbf{U} \cdot \nabla \mathbf{U}^{\dagger}+f \mathbf{R} \mathbf{U}^{\dagger}=-\nabla^{\dagger} p-N \mathbf{S} \mathbf{U}^{\dagger}, \nabla \cdot \mathbf{U}=0
$$

where $\nabla^{\dagger} p=(\nabla p, 0)$ and time was non-dimensionalized using turbulence time scale $L / U_{h}$. Here

$$
\mathbf{R}=\left(\begin{array}{ll}
\mathbf{J} & 0 \\
\mathbf{0} & 0
\end{array}\right), \mathbf{S}=\left(\begin{array}{ll}
\mathbf{0} & \mathbf{0} \\
\mathbf{0} & \mathbf{J}
\end{array}\right), \mathbf{J}=\left(\begin{array}{cc}
0 & -1 \\
1 & 0
\end{array}\right),
$$

and $\mathbf{R}_{n}, \mathbf{S}_{n}$ will denote the action of $\mathbf{R}$ and $\mathbf{S}$ on $n$-th Fourier component, $\mathbf{M}=f \mathbf{R}+N \mathbf{S}$. In this section we write Boussinesq equations in the Craya-Herring cyclic basis and use this representation to perform time averaging. Linear equations describing inertio-gravity waves are

$$
\partial_{t} \mathbf{U}^{\dagger}+\mathbf{M U}^{\dagger}=-\nabla^{\dagger} p, \nabla \cdot \mathbf{U}=0 .
$$

Dispersion relation for inertio-gravity waves which are solutions of Eqs. (2.3) has the form

$$
\omega_{n}^{2}=N^{2} \frac{\left|\check{n}^{\prime}\right|^{2}}{|\check{n}|^{2}}+f^{2} \frac{n_{3}^{2} / a_{3}^{2}}{|\check{n}|^{2}}=R o_{a}^{-2}\left(B u \frac{\left|\check{n}^{\prime}\right|^{2}}{|\check{n}|^{2}}+\frac{n_{3}^{2}}{|\check{n}|^{2}}\right)=N^{2}\left(\frac{\left|\check{n}^{\prime}\right|^{2}}{|\check{n}|^{2}}+B u^{-1} \frac{n_{3}^{2}}{|\check{n}|^{2}}\right)
$$

where $|\check{n}|^{2}=n_{1}^{2}+n_{2}^{2} / a_{2}^{2}+n_{3}^{2} / a_{3}^{2},\left|\breve{n}^{\prime}\right|^{2}=n_{1}^{2}+n_{2}^{2} / a_{2}^{2}$. Here $R o_{a}$ is the anisotropic Rossby number and $B u$ is the Burger number defined by (1.4), (1.5). It follows from (2.4) that the effect of rotation and stratification are not uniform on scales. In the case $\left|\check{n}^{\prime}\right| /\left|n_{3}\right|>>1$ gravity waves are fast and inertial waves are slow. On the other hand, for scales satisfying $\left|n_{3}\right| /\left|\check{n}^{\prime}\right|<<1$ gravity waves are slow and inertial waves are fast. Control of resonances coupling fast waves on small scales and fast waves on large scales can only be achieved through a careful analysis of small divisors in resonances (BMN, 1996 a, b, d and Section 6).

The inertio-gravity wave propagator is the operator solution $\mathbf{E}(t)(\mathbf{E}(0)=\mathbf{I} \mathbf{d}$ is the identity) to the linear problem (2.3):

$$
\Phi(t)=\mathbf{E}(t) \Phi_{0} ; \quad \Phi(0)=\Phi_{0}
$$


where $\Phi(t)=(\mathbf{U}(t), \rho(t))$. The operator $\mathbf{E}(t)$ describes propagation of inertio-gravity waves. In the CrayaHerring cyclic basis (Riley et al. (1981), Métais and Herring (1989), Godeferd and Cambon (1994) present developments in this basis for stratified flows without rotation) linear problem (2.3) restricted on the subspace of divergence free vector fields reduces to the following $3 \times 3$ matrix for the $n$-th Fourier component:

$$
\omega_{n} \mathbf{M}^{\prime}=\omega_{n}\left(\begin{array}{ccc}
0 & 0 & 0 \\
0 & 0 & -1 \\
0 & 1 & 0
\end{array}\right)
$$

where zero eigenvalue corresponds to the quasi-geostrophic mode. We use the extended notation

$$
\check{m}=\left[m_{1}, m_{2} / a_{2}, m_{3} / a_{3}, 0\right], 0<a_{2} \leq 1,
$$

and similarly for $n, k$. We introduce the orthonormal basis of the divergence-free subspace for $n$-th Fourier mode:

$$
p_{0 n}=\left[-\frac{\check{n}_{2}}{\left|\check{n}^{\prime}\right|}, \frac{n_{1}}{\left|\check{n}^{\prime}\right|}, 0,0\right] ; p_{1 n}=\left[\frac{n_{1} \check{n}_{3}}{|\check{n}|\left|\check{n}^{\prime}\right|}, \frac{\check{n}_{2} \check{n}_{3}}{|\check{n}|\left|\check{n}^{\prime}\right|}, \frac{-n_{1}^{2}-\check{n}_{2}^{2}}{|\check{n}|\left|\check{n}^{\prime}\right|}, 0\right] ; p_{2 n}=e_{4}=[0,0,0,1] .
$$

The vectors $p_{0 k}, p_{0 m}, p_{1 k}$ etc. are defined similarly. The vectors $p_{0 k}, p_{1 k}, p_{2 k}$ are orthonormal cyclic vectors for the matrix $\mathbf{S}$ restricted on the divergence free Fourier subspace; let $\mathbf{P}_{d}$ be the projection on divergence free vectors in the Helmholtz decomposition (for the velocity component):

$$
\mathbf{P}_{d} \mathbf{S}_{n} p_{0 n}=0 ; \mathbf{P}_{d} \mathbf{S}_{n} p_{1 n}=-\phi_{n} p_{2 n} ; \mathbf{P}_{d} \mathbf{S}_{n} p_{2 n}=\phi_{n} p_{1 n} ; \phi_{n}=\frac{\left|\check{n}^{\prime}\right|}{|\check{n}|} .
$$

The $p_{j n}$ are the Craya-Herring basis for the purely stratified problem, already used by Riley et al. (1981). In the case $f \neq 0$ we use the following orthonormal basis

$$
q_{0 n}=\frac{1}{\omega_{n}}\left(N \phi_{n} p_{0 n}+f \xi_{n} p_{2 n}\right), q_{1 n}=p_{1 n}, q_{2 n}=\frac{1}{\omega_{n}}\left(N \phi_{n} p_{2 n}-f \xi_{n} p_{0 n}\right)
$$

where

$$
\xi_{n}=\frac{\check{n}_{3}}{|\check{n}|}, \omega_{n}^{2}=N^{2} \phi_{n}^{2}+f^{2} \xi_{n}^{2}
$$

The vectors $q_{0 n}, q_{1 n}$ and $q_{2 n}$ are orthonormal and form a basis in the space of divergence free vector fields. In the case $n_{1}=n_{2}=0$ (this case corresponds to taking horizontal averages) we have from (2.9) (we have used the fact that $\left.\left.p_{1 n}\right|_{n_{1}=n_{2}=0}=\left(\frac{1}{\sqrt{2}}, \frac{1}{\sqrt{2}}, 0,0\right)\right)$

$$
q_{0 \bar{n}}=(0,0,0,1) ; q_{1 \bar{n}}=\left(\frac{1}{\sqrt{2}}, \frac{1}{\sqrt{2}}, 0,0\right) ; q_{2 \bar{n}}=\left(\frac{1}{\sqrt{2}},-\frac{1}{\sqrt{2}}, 0,0\right)
$$

where $\bar{n}=\left(0,0, n_{3}\right)$ denotes wavenumbers for which $n_{1}=n_{2}=0$. In this paper we use the overbar notation for the operation of horizontal averaging

$$
\bar{F}\left(t, x_{3}\right)=\frac{1}{2 \pi 2 \pi / a_{2}} \int_{0}^{2 \pi} \int_{0}^{2 \pi / a_{2}} F\left(t, x_{1}, x_{2}, x_{3}\right) d x_{1} d x_{2} .
$$


For the matrix of linear problem

$$
\mathbf{M}_{n}=N \mathbf{S}_{n}+f \mathbf{R}_{n}
$$

the vectors (2.9) form a cyclic basis since, after projection on divergence-free vector fields via Helmholtz decomposition:

$$
\mathbf{P}_{d} \mathbf{M} q_{0 n}=0, \mathbf{P}_{d} \mathbf{M} q_{1 n}=-\omega_{n} q_{2 n}, \mathbf{P}_{d} \mathbf{M} q_{2 n}=\omega_{n} q_{1 n}
$$

and, as $\omega_{n}^{2}=f^{2}$ for $n_{1}=n_{2}=0$

$$
\mathbf{P}_{d} \mathbf{M} q_{0 \bar{n}}=0, \mathbf{P}_{d} \mathbf{M} q_{1 \bar{n}}=-f q_{2 \bar{n}}, \mathbf{P}_{d} \mathbf{M} q_{2 \bar{n}}=f q_{1 \bar{n}}
$$

Any arbitrary divergence-free vector field $\mathbf{U}_{n}^{\dagger}$ can be written as

$$
\mathbf{U}_{n}^{\dagger}=V_{n}^{0} q_{0 n}+V_{n}^{1} q_{1 n}+V_{n}^{2} q_{2 n} .
$$

We shall use the variables $V$ to denote vector of coefficients corresponding to $\mathbf{U}_{n}^{\dagger}: \mathbf{V}_{n}=\left[V_{n}^{0}, V_{n}^{1}, V_{n}^{2}\right]=$ $\left[V_{n}^{0}, \mathbf{V}_{n}^{\prime}\right], \mathbf{V}_{n}^{\prime}=\left[V_{n}^{1}, V_{n}^{2}\right]$. We denote by $\Pi_{n}^{Q G}$ the projection onto $q_{0 n}$ (quasi-geostrophic mode):

$$
\Pi^{Q G} \mathbf{U}^{\dagger}(x)=\sum_{n} V_{n}^{0} q_{0 n} e^{i n \cdot x}, \Pi_{n}^{Q G} \mathbf{U}_{n}^{\dagger}=V_{n}^{0} q_{0 n} .
$$

Similarly, we define the projection $\Pi^{A G}$ onto ageostrophic component:

$$
\Pi^{A G} \mathbf{U}^{\dagger}(x)=\sum_{n}\left(V_{n}^{1} q_{1 n}+V_{n}^{2} q_{2 n}\right) e^{i n \cdot x}, \Pi_{n}^{A G} \mathbf{U}_{n}^{\dagger}=V_{n}^{1} q_{1 n}+V_{n}^{2} q_{2 n} .
$$

The action of the linear propagator on the Fourier components $\mathbf{E}(t)$ can be written in $V$-variables

$$
\mathbf{E}(t)\left[V^{0}, \mathbf{V}^{\prime}\right]_{n}=\exp \left(\omega_{n} t \mathbf{M}_{n}^{\prime}\right)\left[V^{0}, \mathbf{V}^{\prime}\right]_{n}=\left[V^{0}, \exp \left(\omega_{n} t \mathbf{J}\right) \mathbf{V}^{\prime}\right]
$$

Here $\mathbf{J}, \mathbf{M}_{n}^{\prime}$ are given by

$$
\mathbf{M}_{n}^{\prime}=\left(\begin{array}{ccc}
0 & 0 & 0 \\
0 & 0 & -1 \\
0 & 1 & 0
\end{array}\right), \quad \mathbf{J}=\left(\begin{array}{cc}
0 & -1 \\
1 & 0
\end{array}\right)
$$

We have

$$
\exp \left(\omega_{n} \mathbf{J} t\right)=\cos \left(\omega_{n} t\right) \mathbf{I}+\sin \left(\omega_{n} t\right) \mathbf{J}
$$

Obviously, $\mathbf{E}(t)$ represents vector rotation in $V^{1}, V^{2}$-plane; $V^{0}$ component (called 3DQG) is not affected. Note that the relation between $\mathbf{U}$ and $\mathbf{V}$ variables is given by

$$
V_{n}^{0}=\mathbf{U}_{n}^{\dagger} \cdot q_{0 n}, V_{n}^{1}=\mathbf{U}_{n}^{\dagger} \cdot q_{1 n}, V_{n}^{2}=\mathbf{U}_{n}^{\dagger} \cdot q_{2 n} .
$$


Clearly, $V_{n}^{0^{*}}=-V_{-n}^{0}$ for real $\mathrm{U}(x)$.

The Boussinesq equations (2.1) in Fourier representation in $V$ variables can be written in the cyclic basis (2.9) as

$$
\partial_{t} V_{n}^{i_{3}}=-i \sum_{k+m=n, i_{1}, i_{2}} Q_{k m n}^{i_{1} i_{2} i_{3}} V_{k}^{i_{1}} V_{m}^{i_{2}}-\omega_{n}\left(M_{n}^{\prime} V_{n}\right)^{i_{3}}
$$

where $i_{1}, i_{2}, i_{3}=0,1,2, M^{\prime}$ is the matrix $M$ in $V$-variables given by (2.18). The coefficients $Q_{k m n}^{i_{1} i_{2} i_{3}}$ are determined from the equations

$$
Q_{k m n}^{i_{1} i_{2} i_{3}}=\left(q_{i_{1} k} \cdot m\right)\left(q_{i_{2} m} \cdot q_{i_{3} n}\right)
$$

The coefficients $Q_{k m n}^{i_{1} i_{2} i_{3}}, k+m=n$, are explicitly given by a straightforward computation using (2.9), (2.22). For the coefficient describing interaction of 0 -modes (quasi-geostrophic) we obtain $\left(n^{\prime} \wedge m^{\prime} \equiv n_{1} m_{2}-n_{2} m_{1}\right)$.

$$
Q_{k m n}^{000}=\frac{N \check{n}^{\prime} \wedge \check{m}^{\prime}}{\omega_{k} \omega_{m} \omega_{n}|\grave{k} \| \check{m}||\check{n}|}\left(N^{2} \check{n}^{\prime} \cdot \check{m}^{\prime}+f^{2} \check{n}_{3} \check{m}_{3}\right) .
$$

Since skew-symmetric in $k, m$ component of $Q_{k m n}^{000}$ makes no contribution to Eq. $(2.21)$, we can use the following $Q_{k m n}^{000}$ in Eq. (2.21):

$$
Q_{k m n}^{000}=\frac{N \check{k}^{\prime} \wedge \check{m}^{\prime}}{\omega_{k} \omega_{m} \omega_{n}|\ddot{k} \| \check{m}||\check{n}|}\left(N^{2}\left|\check{m}^{\prime}\right|^{2}+f^{2} \check{m}_{3}^{2}\right)=\frac{N \omega_{m}|\check{m}| \check{k}^{\prime} \wedge \check{m}^{\prime}}{\omega_{k} \omega_{n}|\check{k}||\check{n}|}
$$

where we have used $|\check{m}|^{2} \omega_{m}^{2}=N^{2}\left|\check{m}^{\prime}\right|^{2}+f^{2} \check{m}_{3}^{2}$. The coefficients $Q_{k m n}^{000}$ given by (2.24) is the familiar coefficient in 3DQG equations written in cyclic basis. Other coefficients in (2.21) have the form

$$
\begin{aligned}
& Q_{k m n}^{012}=-\frac{f N \xi_{m} \xi_{n}\left(\check{k}^{\prime} \wedge \check{m}^{\prime}\right)^{2}}{\omega_{k} \omega_{n}|\breve{k}|\left|\check{m}^{\prime}\right|\left|\check{n}^{\prime}\right|}, Q_{m k n}^{102}=-\frac{f N\left(\check{m}_{3} \check{k}^{\prime} \cdot \check{m}^{\prime}-\breve{k}_{3}\left|\check{m}^{\prime}\right|^{2}\right)\left(\check{n}_{3} \check{n}^{\prime} \cdot \breve{k}^{\prime}-\breve{k}_{3}\left|\check{n}^{\prime}\right|^{2}\right)}{\omega_{k} \omega_{n}|\check{k}|\left|\check{m}_{\|}\right||\check{n}|\left|\check{m}^{\prime}\right|\left|\check{n}^{\prime}\right|} \\
& Q_{k m n}^{021}=\frac{f N \xi_{m} \xi_{n}\left(\check{k}^{\prime} \wedge \check{m}^{\prime}\right)^{2}}{\omega_{k} \omega_{m}|\breve{k}|\left|\check{m}^{\prime}\right|\left|\check{n}^{\prime}\right|}, Q_{m k n}^{201}=\frac{f N \xi_{m} \xi_{n}\left(\check{k}^{\prime} \wedge \check{m}^{\prime}\right)^{2}}{\omega_{k} \omega_{m}|\check{k}|\left|\check{m}^{\prime}\right|\left|\check{n}^{\prime}\right|} \\
& Q_{k m n}^{011}=\frac{N\left(\check{k}^{\prime} \wedge \check{m}^{\prime}\right)\left(\check{n}_{3} \check{m}_{3} \check{n}^{\prime} \cdot \check{m}^{\prime}+\left|\check{n}^{\prime}\right|^{2}\left|\check{m}^{\prime}\right|^{2}\right)}{\omega_{k}\left|\check{m}^{\prime}\right|\left|\check{n}^{\prime}\right||\check{k}\|\check{m}\| \check{n}|}, Q_{m k n}^{101}=\frac{N \xi_{n}\left(\check{k}^{\prime} \wedge \check{m}^{\prime}\right)\left(\check{m}_{3} \check{k}^{\prime} \cdot \check{m}^{\prime}-\check{k}_{3}\left|\check{m}^{\prime}\right|^{2}\right)}{\omega_{k}\left|\check{k}_{\|}\right|\left|\check{m}_{\|}\right|\left|\check{m}^{\prime}\right|\left|\check{n}^{\prime}\right|}, \\
& Q_{k m n}^{022}=\frac{N\left(\check{k}^{\prime} \wedge \check{m}^{\prime}\right)\left(N^{2}\left|\check{m}^{\prime}\right|^{2}\left|\check{n}^{\prime}\right|^{2}+f^{2} \check{m}_{3} \check{n}_{3} \check{m}^{\prime} \cdot \check{n}^{\prime}\right)}{\omega_{k} \omega_{m} \omega_{n}|\check{k}||\check{m}||\check{n}|\left|\check{m}^{\prime}\right|\left|\check{n}^{\prime}\right|}, Q_{n k n}^{202}=\frac{f^{2} N \check{m}_{3}\left(\check{k}^{\prime} \wedge \check{m}^{\prime}\right)\left(\check{k}_{3}\left|\check{n}^{\prime}\right|^{2}-\check{n}_{3} \check{n}^{\prime} \cdot \check{k}^{\prime}\right)}{\omega_{k} \omega_{m} \omega_{n}|\check{k}||\check{m}||\check{n}|\left|\check{m}^{\prime}\right|\left|\check{n}^{\prime}\right|}
\end{aligned}
$$

In addition, we have coefficients associated with the operation of horizontal averaging, where $\bar{k}=\left(0,0, k_{3}\right)$ :

$$
Q_{\bar{k} m n}^{000}=Q_{k \bar{m} n}^{000}=Q_{k m \bar{n}}^{000}=0 ; Q_{\bar{k} m n}^{012}=Q_{\bar{k} m n}^{021}=Q_{\bar{k} m n}^{011}=Q_{\bar{k} m n}^{022}=0
$$


Also, $Q_{\bar{k} \dot{m} n}^{i_{1} i_{2} 0}=0\left(i_{1} \neq 0, i_{2} \neq 0\right)$ and

$$
Q_{m \bar{k} n}^{102}=-\frac{N \phi_{m} \phi_{n}}{\omega_{n}} \breve{k}_{3}, Q_{m \bar{k} n}^{201}=Q_{m \bar{k} n}^{101}=Q_{m \bar{k} n}^{202}=0 .
$$

We note that $V_{k}^{i_{1}} V_{m}^{i_{2}}=V_{m}^{i_{2}} V_{k}^{i_{1}}$ and then collecting terms in Eqs. (2.21) we define the symmetrized

$$
\hat{Q}_{k m n}^{011}=Q_{k m n}^{011}+Q_{m k n}^{101}, \hat{Q}_{k m n}^{012}=Q_{k m n}^{012}+Q_{m k n}^{102}, \hat{Q}_{k m n}^{021}=Q_{k m n}^{021}+Q_{m k n}^{201}, \hat{Q}_{k m n}^{022}=Q_{k m n}^{022}+Q_{m k n}^{202}
$$

It implies that

$$
\hat{Q}_{\bar{k} m n}^{021}=\hat{Q}_{\bar{k} m n}^{011}=\hat{Q}_{\bar{k} m n}^{022}=0, \hat{Q}_{\bar{k} m n}^{012}=-\frac{N \phi_{m} \phi_{n}}{\omega_{n}} \breve{k}_{3} .
$$

\section{The limit equations describing reduced dynamics}

We write classical rotating Boussinesq equations using the variables $V$ in the basis (2.9); these equations have the form (see Eqs. (2.21))

$$
\partial_{t} \mathbf{V}_{n}+\omega_{n} \mathbf{M}_{n}^{\prime} \mathbf{V}_{n}=(\mathbf{B}(\mathbf{V}, \mathbf{V}))_{n}
$$

Here $\mathbf{M}^{\prime}$ is the linear propagator operator corresponding to inertio-gravity waves given in cyclic $V$-variables by Eqs. (2.17)-(2.18). We introduce this linear propagator directly into nonlinearity in (3.1) using the change of variables $\mathbf{V}=\mathbf{E}(-t) \mathbf{v}, \mathbf{V}_{n}=\exp \left(-\omega_{n} \mathbf{M}_{n}^{\prime} t\right) \mathbf{v}_{n}$ where $\mathbf{v}=\left[v^{0}, v^{1}, v^{2}\right]$ and $\mathbf{M}^{\prime}$ is defined by (2.18). Boussinesq equations (3.1) written in $\mathbf{v}$ variables have the form

$$
\partial_{t} \mathbf{v}=\mathbf{B}(t, \mathbf{v}, \mathbf{v}), \mathbf{B}(t, \mathbf{v}, \mathbf{v})=\mathbf{E}(t) \mathbf{B}(\mathbf{E}(-t) \mathbf{v}, \mathbf{E}(-t) \mathbf{v})
$$

Equations (3.2) are explicitly time-dependent with rapidly varying coefficients. The following equations describing reduced dynamics are associated with Eqs. (3.2) (BMN, 1996b):

$$
\partial_{t} \mathbf{w}=\tilde{\mathbf{B}}(\mathbf{w}, \mathbf{w}), \tilde{\mathbf{B}}(\mathbf{w}(t), \mathbf{w}(t))=\lim _{T \rightarrow+\infty} \frac{1}{T} \int_{0}^{T} \mathbf{B}(s, \mathbf{w}(t), \mathbf{w}(t)) d s .
$$

Clearly, when represented in Fourier modes, the right-hand-side of (3.3) will be determined by resonances $\pm \omega_{k} \pm \omega_{m} \pm \omega_{n}=0$ within terms of the type $\exp \left(i\left( \pm \omega_{k} \pm \omega_{m} \pm \omega_{n}\right) s\right)$, see (2.17), (2.19). Projecting Eqs. (3.3) on the 3DQG mode $q_{0 n}$ corresponding to the zero eigenvalue of the linear problem, we find that the limit equations (3.3) describing reduced dynamics contain classical 3DQG equations as a completely decoupled subsystem, a result already published in BMN (1996b). This confirms the QG decoupling of the reduced equations after their projection on the mode corresponding to the zero eigenvalue which was also proven for the rotating shallow-water equations and 3D rotating Euler equations (Mahalov and Marcus, 1995; BMN, 1995; BMN, 1996a; BMN, 1996b). In the case of 3D rotating Euler the reduced equations were projected 
on the subspace of $2 \mathrm{D}-3 \mathrm{C}$ fields by means of the operation of vertical averaging. Two-dimensional, three components refer to fields that have three components and depend on two variables. These are MFI (material frame indifferent fields) uneffected by rotation (Ristorcelli et al., 1995; Speziale, 1989). In the case of rotating shallow-water equations reduced equations were projected on the quasi-geostrophic mode and decoupling of quasi-geostrophic equations was obtained and published in Mahalov and Marcus (1995) before Embid and Majda (1996). In the important paper by Warn (1986) the same result for shallow-water equations had been obtained based on formal two time scales expansions and Ertel's theorem. To prove the decoupling for Boussinesq equations, Embid and Majda (1996) also used Ertel's theorem, which does not give insight into the structure of the ageostrophic dynamics in the context of Boussinesq equations.

Now we ouline a new proof of decoupling of 3DQG equations in (3.3) We refer to BMN $(1996 \mathbf{a}, \mathrm{b}, \mathrm{c})$ and Mahalov and Marcus (1995) for detailed proofs of splitting in Boussinesq, 3D Euler and rotating shallowwater equations. By (3.2) and (3.3) the only active triadic interactions in the reduced equations (3.3) are those satisfying the relation $\pm \omega_{k} \pm \omega_{m} \pm \omega_{n}=0$. We note that projection of Eqs. (3.3) on QG mode (which corresponds to zero eigenvalue of the linear problem) leads to the additional constraint $\omega_{n}=0$. Then the conditions $\pm \omega_{k} \pm \omega_{m} \pm \omega_{n}=0$ and $\omega_{n}=0$ reduce to 2-wave interactions $\omega_{k}=\omega_{m}$. For $N^{2} \neq f^{2}$ the condition $\omega_{k}=\omega_{m}$ is equivalent to the condition $\left|\breve{k}^{\prime}\right| /|\breve{k}|=\left|\check{m}^{\prime}\right| /|\check{m}|$ (equivalently, $\phi_{k}=\phi_{m}$; see (2.8)). Clearly, reduced equations (3.3) projected on $Q G$ mode involve only the coefficients $Q_{k m n}^{i_{1} i_{2} i_{3}}$ with $i_{3}=0$ $(n=k+m)$. One trivial solution of $\omega_{k}=\omega_{m}$ is $\omega_{k}=\omega_{m}=0$ which corresponds to the QG coefficient $Q_{k m n}^{000}$. An important observation is that other terms involving the coefficients $Q_{k m n}^{i_{1} i_{2} 0}\left(i_{1} \neq 0\right.$ or $\left.i_{2} \neq 0\right)$ in Eqs. (3.3) are annihilated when the resonance condition $\phi_{k}=\phi_{m}$ is used. For the Boussinesq case, the detailed proof can be found in BMN (1996b).

Thus, in the reduced equations (3.3) the total velocity splits into the limit quasi-geostrophic field $\tilde{\mathbf{U}}^{\dagger}\left(t, x_{1}, x_{2}, x_{3}\right)$ satisfying (3.12) and ageostrophic component satisfying equations of the type:

$$
\partial_{t} \mathbf{w}_{A G}=\mathbf{B}_{2}\left(\tilde{\mathbf{U}}^{\dagger}(t), \mathbf{w}_{A G}\right)+\mathbf{B}_{3}\left(\mathbf{w}_{A G}, \mathbf{w}_{A G}\right)
$$

In this paper we are interested in the situation when the quasi-geostrophic (balanced) field is present and it is dominant. We explore the implications of the existence of the vortical mode (at mesoscales the vortical mode represents quasi-geostrophic flows) on the dynamics. The major shortcoming of many existing theories is that they neglect the interaction with the vortical (=potential vorticity carrying) mode of motion (Müller et al., 1986). The results of the study by Lelong and Riley (1991) provide further evidence that the role of the vortical mode in influencing the evolution of strongly stratified flows may be significant and should not be neglected. According to Müller et al. (1986), interactions among internal waves and vortical modes loom as one of the important questions to be addressed by a strong interaction theory. In BMN (1996 a-e) 
and here we present such strong interaction theory based on rigorous KAM type mathematical analysis and small divisor estimates.

In BMN (1996b) and in Section 6 it is shown that the main contribution to resonances in the reduced equations (3.4) is given by the term $\mathbf{B}_{2}\left(\tilde{\mathbf{U}}^{\dagger}(t), \mathbf{w}_{A G}\right)$. We arrive at the same conclusion empirically comparing orders of magnitude of the term $\mathbf{B}_{2}\left(\tilde{\mathbf{U}}^{\dagger}(t), \mathbf{w}_{A G}\right)$ and $\mathbf{B}_{3}\left(\mathbf{w}_{A G}, \mathbf{w}_{A G}\right)$ :

$$
\frac{\left|\mathbf{B}_{3}\left(\mathbf{w}_{A G}, \mathbf{w}_{A G}\right)\right|}{\left|\mathbf{B}_{2}\left(\tilde{\mathbf{U}}^{\dagger}(t), \mathbf{w}_{A G}\right)\right|} \approx \frac{\left|\mathbf{w}_{A G}\right|}{\left|\tilde{\mathbf{U}}^{\dagger}\right|} \approx 0.1
$$

for synoptic scales at midlatitudes (e.g. Holton, 1992). Then equations become linear in $\mathbf{w}_{A G}$ with coefficients depending on arbitrary quasi-geostrophic field $\tilde{\mathbf{U}}^{\dagger}(t)$ :

$$
\partial_{t} \mathbf{w}_{A G}=\mathbf{B}_{2}\left(\tilde{\mathbf{U}}^{\dagger}(t), \mathbf{w}_{A G}\right) .
$$

The numerical work of Bartello (1996) also shows that resonant 3-wave interactions are of secondary importance in the overall picture of interactions when both rotation and stratification are present and $B u=O(1)$. They do not lead to slow-fast energy exchange and are difficult to resonate. Rigorous proof of this result was given in Babin, Mahalov and Nicolaenko (1996b) using small divisor estimates and is discussed in Section 6. Geostrophic adjustment takes place via catalytic interaction involving a "rotational" ("vortical") mode and two inertial-gravity modes. As in Bartello (1995), an interaction is "catalytic" in that it does not influence slow modes, but serves to transfer fast AG energy downscale.

The bilinear form $\tilde{\mathbf{B}}(\mathbf{w}, \mathbf{w})$ in Eqs. (3.3) can be conveniently represented in the cyclic basis (2.9). Now we write the resonant (limit) equation for $\mathbf{w}=w^{0} q_{0}+w^{1} q_{1}+w^{2} q_{2}$ where $w^{0}$ is simply projection of $\mathbf{w}$ on QG mode. Then $w^{0}$ can be defined from the quasi-geostrophic equation; $\mathbf{w}^{\prime}=\left(w^{1}, w^{2}\right)$ is found from inertio-gravity wave limit equation. The quasi-geostrophic equation is given by

$$
\partial_{t} w_{n}^{0}=\mathbf{B}_{1}\left(w^{0}, w^{0}\right)_{n}, \mathbf{B}_{1}\left(w^{0}, w^{0}\right)_{n}=-i \sum_{k+m=n} Q_{k m n}^{000} w_{k}^{0} w_{m}^{0}, Q_{k m n}^{000}=\frac{N \omega_{m}|\check{m}| \check{k}^{\prime} \wedge \check{m}^{\prime}}{\omega_{k} \omega_{n}|\check{k}||\check{n}|}
$$

and in the case $n_{1}=n_{2}=0$

$$
\mathbf{B}_{1}\left(w^{0}, w^{0}\right)_{\bar{n}}=-i \sum_{k+m=n} Q_{k m \bar{n}}^{000} w_{k}^{0} w_{m}^{0}=0
$$

since $Q_{k m \bar{n}}^{000}=0$ by (2.29). From (2.29), we also prove that $\bar{\rho}$ and the horizontal averages of velocities do not enter the QG equation (3.7); that there are no $\mathbf{w}_{\bar{k}}^{0}$ or $\mathbf{w}_{\bar{m}}^{0}$. This is not true for the operator splitting in the limit $f=0, F_{h}<<1, B u>>1$.

We introduce variables $\tilde{q}, \tilde{\mathbf{U}}$ (quasi-geostrophic potential and velocity, not to be confused with the cyclic basis vectors $q_{0 n}, q_{1 n}$ and $\left.q_{2 n}\right)$ :

$$
\tilde{q}_{m}=\omega_{m}|\check{m}| w_{m}^{0}, \quad \tilde{\mathbf{U}}_{k}=N\left[-k_{2} / a_{2}, k_{1}, 0,0\right] \tilde{q}_{k} / \omega_{k}^{2}|\check{k}|^{2} .
$$


The quasi-geostrophic stream function is defined by $\tilde{\psi}_{k}=\tilde{q}_{k} N / \omega_{k}^{2}|\breve{k}|^{2}$. Recalling that $\omega_{k}^{2}|\breve{k}|^{2} / N=\left|\breve{k}^{\prime}\right|^{2}+\mu^{2} \breve{k}_{3}^{2}$, $\mu=f / N$ we find a familiar formula which relates $\tilde{\psi}$ and $\tilde{q}$ in physical space

$$
-\left(\nabla_{h}^{2}+\mu^{2} \frac{\partial^{2}}{\partial x_{3}^{2}}\right) \tilde{\psi}=\tilde{q}
$$

Using (3.9), Eq. (3.7) is written in the form:

$$
\partial_{t} \tilde{q}_{n}=-i \sum_{k+m=n}\left(\tilde{\mathbf{U}}_{k} \cdot m\right) \tilde{q}_{m}
$$

$\tilde{q}(t, x)$ obeys in physical space the 3D quasi-geostrophic equations (Bourgeois and Beale, 1994)

$$
\frac{\tilde{D}}{\tilde{D} t} \tilde{q}=0
$$

where $\frac{\tilde{D}}{\tilde{D} t}$ is the advective derivative, based on the quasi-geostrophic velocity and $\tilde{q}$ is the quasi-geostrophic potential.

Now we describe adiabatic invariants associated with horizontal averaging. Let us denote $\bar{n}$ as $\left(0,0, n_{3}\right)$. We note that in the case $n_{1}=n_{2}=0$ we have $q_{0 \bar{n}}=[0,0,0,1]$ and, therefore, $w_{\bar{n}}^{0}=\rho_{\bar{n}}$. Now the fact that $Q_{k m \bar{n}}^{i_{1} i_{2} 0}=0$ implies $\frac{\partial}{\partial t} \rho_{\bar{n}}=0$. Then horizontal averages of $\rho$ are conserved by the reduced equations. We outline the proof of this important new result, that is $\frac{\partial}{\partial t} \rho_{\bar{n}}=0$. First notice that $Q_{k m \bar{n}}^{000}=0$ from $(2.23)$. Also, $Q_{k m \bar{n}}^{110}=Q_{k m \bar{n}}^{220}=Q_{k m \bar{n}}^{210}=0$. Then $\rho_{\bar{n}}$, the $n$-th Fourier coefficient of $\bar{\rho}\left(t, x_{3}\right)$ satisfies:

$$
\frac{\partial}{\partial t} \rho_{\bar{n}}=\sum_{k+m=n, n^{\prime}=0} Q_{k m \bar{n}}^{120}\left(w_{k}^{1} w_{m}^{2}-w_{k}^{2} w_{m}^{1}\right)
$$

the right hand side is null on 2-wave interactions $\phi_{k}=\phi_{m}$ with $n^{\prime}=0$. Clearly, in (3.13) no 3 wave resonances are omitted. This result holds for all resonances. The proof is very closely related to the one for exact QG splitting (see discussion before Eq. (3.4)) but does not follow immediately from Ertel's theorem. This exact conservation law of the reduced equations correspond to adiabatic invariant of the full Boussinesq equations. In particular, it shows that in the asymptotic state (after several periods of oscillations associated with wave motions) horizontally averaged buoyancy variable will reach a constant in time value. It confirms that horizontal spatial averages of buoyancy variable $\bar{\rho}\left(x_{3}\right)$ are time independent in the asymptotic state; this is frequently done in many investigations addressing the impact of vertical variability of buoyancy on the dynamics (e.g. Howard, 1972; McWilliams and Weiss, 1996; Doering and Constantin, 1996). Below we find the impact of buoyancy phases associated with vertical shearing of this invariant on ageostrophic dynamics in non-hydrostatic situation. Similarly, we prove that $\bar{U}^{1}, \bar{U}^{2}$ undergo rigid $\Omega$-rotation in the $B u=O(1)$ limit (this result is true for all resonances including 3 wave resonances) and they are true adiabatic invariants only if $\Omega=0$. 
The limit equations for $\mathbf{w}_{n}^{\prime}=\left[w_{n}^{1}, w_{n}^{2}\right]$ include $w_{m}^{1}, w_{m}^{2}$ and already found $w_{k}^{0}$. Therefore, taking into account $k, m$-symmetry, we always label $w^{0}$ variable by $k$; therefore to write the resonant equations it suffices to consider $\hat{Q}_{k m n}^{011}, \hat{Q}_{k m n}^{012}, \hat{Q}_{k m n}^{021}, \hat{Q}_{k m n}^{022}$ (recall that all 1-wave and 3-wave interactions are non-resonant, cf. Section 6). The resonant relations reduce to $\omega_{n}=\omega_{m}, n=k+m$ for the "symmetrized" $\hat{Q}_{k m n}^{i_{1} i_{2} i_{3}}$ defined in (2.31). The original Boussinesq equations for $\mathbf{v}^{\prime}=\Pi^{A G} \mathbf{v}$ can be written in the form

$$
\partial_{t} \mathbf{v}_{n}^{\prime}=-i \exp \left(\omega_{n} t \mathbf{J}\right) \sum_{k+m=n} v_{k}^{0} \hat{\mathbf{Q}}_{k m n} \exp \left(-\omega_{m} t \mathbf{J}\right) \mathbf{v}_{m}^{\prime}+\Pi_{n}^{A G} \hat{\mathbf{Q}}^{+}(N t, \mathbf{v})_{n}
$$

Here the matrix $\hat{\mathbf{Q}}_{k m n}$ is defined by

$$
\hat{\mathbf{Q}}_{k m n}=\left(\begin{array}{cc}
\hat{Q}_{k m n}^{011} & \hat{Q}_{k m n}^{021} \\
\hat{Q}_{k m n}^{012} & \hat{Q}_{k m n}^{022}
\end{array}\right)
$$

$\hat{Q}_{k m n}^{011}$ etc. are given above in (2.31)-(2.32), $\mathbf{J}$ is given by $(2.18) ; \hat{\mathbf{Q}}^{+}(N t, \mathbf{v})_{n}$ are non-resonant terms. Taking into account (2.19), we see that resonant interactions are generated by squares of sines and cosines and are given by the matrix $\tilde{\mathbf{Q}}=(\hat{\mathbf{Q}}-\mathbf{J} \hat{\mathbf{Q}} \mathbf{J}) / 2$, namely:

$$
\tilde{\mathbf{Q}}_{k m n}=\left(\begin{array}{cc}
\hat{Q}_{k m n}^{011}+\hat{Q}_{k m n}^{022}, & -\hat{Q}_{k m n}^{012}+\hat{Q}_{k m n}^{021} \\
-\hat{Q}_{k m n}^{021}+\hat{Q}_{k m n}^{012}, & \hat{Q}_{k m n}^{011}+\hat{Q}_{k m n}^{022}
\end{array}\right) .
$$

Computing explicitly resonant terms in (3.14) we obtain reduced resonant equations for the limit $\mathbf{w}$, with $\mathbf{w}_{n}^{\prime}=\left(w_{n}^{1}, w_{n}^{2}\right)$

$$
\partial_{t} \mathbf{w}_{n}^{\prime}=i \sum_{\substack{\omega_{m}=\omega_{n} \\ k+m=n, k^{\prime}=0}} \frac{N \phi_{m} \phi_{n}}{\omega_{n}} \breve{k}_{3} \bar{\rho}_{k} \mathbf{J} \mathbf{w}_{m}^{\prime}-i \sum_{\substack{\omega_{m}=\omega_{n} \\ k+m=n, k^{\prime} \neq 0}} w_{k}^{0}(t) \tilde{\mathbf{Q}}_{k m n} \mathbf{w}_{m}^{\prime} .
$$

We recall that the resonance condition $\omega_{m}=\omega_{n}$ is equivalent to the condition $\frac{\left|\tilde{m}^{\prime}\right|}{\left|\ddot{m}^{\prime}\right|}=\frac{\left|\tilde{n}^{\prime}\right|}{|\bar{n}|}$ (or $\phi_{m}=\phi_{n}$ ) and $\frac{\left|m_{3}\right|}{|\tilde{n}|}=\frac{\left|n_{3}\right|}{|\tilde{n}|}$ (or $\left|\xi_{m}\right|=\left|\xi_{n}\right|$, see (2.10)). In Eqs. (3.17) $w^{0}(t)$ is an arbitrary solution of quasi-geostrophic equation with initial data projected on QG fields and $\tilde{\mathbf{Q}}_{k m n}$ are geometric coefficients given above.

In Eqs. (3.17) $\frac{N \phi_{m} \phi_{n}}{\omega_{n}} \breve{k}_{3} \bar{\rho}_{k}$ represents phase correction impact of vertical shearing of $\bar{\rho}_{k}$ on ageostrophic dynamics. Clearly, $\left(\frac{\partial}{\partial x_{3}} \bar{\rho}\right)_{\bar{k}}=i \breve{k}_{3} \rho_{\bar{k}}$. This phase correction implicit in (3.17) is equivalent to the spectral Doppler phase shift. It can be calculated explicitly as a solution of the linear problem:

$$
\partial_{t} \mathbf{w}_{n}^{\prime}=i \sum_{\substack{\phi_{m}=\phi_{n} \\ k+m=n, k^{\prime}=0}} \frac{N \phi_{m} \phi_{n}}{\omega_{n}} \check{k}_{3} \bar{\rho}_{k} \mathbf{J} \mathbf{w}_{m}^{\prime}
$$

In particular, in (3.18) for all $a_{2}, a_{3}$ the condition $\phi_{m}=\phi_{n}$ is equivalent to $m_{3}=-n_{3}, m^{\prime}=n^{\prime}$ (for $m=n$, $\bar{\rho}_{k}=0$ ). It implies that $k_{3}=2 n_{3}, \phi_{m} \phi_{n}=\phi_{n}^{2}$ in (3.18). With the above remark on the convolution in 
(3.18), Eq. (3.18) reduces to the system coupling $\left(n^{\prime}, n_{3}\right)$ and $\left(n^{\prime},-n_{3}\right)$ :

$$
\partial_{t} \mathbf{w}_{\left(n^{\prime}, \pm n_{3}\right)}^{\prime}= \pm i 2 \check{n}_{3} \bar{\rho}\left(0,0, \pm 2 n_{3}\right) \frac{N \phi_{n}^{2}}{\omega_{n}} \mathbf{J w}_{\left(n^{\prime}, \mp n_{3}\right)}^{\prime}
$$

whose fundamental solution involves $\cos \left(\vartheta_{n} t\right)$ and $\sin \left(\vartheta_{n} t\right)$ with the spectral phase $\vartheta_{n}$ :

$$
\vartheta_{n}^{2}=4 \breve{n}_{3}^{2}\left|\bar{\rho}\left(0,0,2 n_{3}\right)\right|^{2}\left(\frac{N \phi_{n}^{2}}{\omega_{n}}\right)^{2} .
$$

Then in this particular case solutions of (3.17) have the integrating phase factor

$$
\exp \left( \pm i \frac{N \phi_{n}^{2}}{\omega_{n}} 2 \check{n}_{3}\left|\rho_{\left(0,0,2 n_{3}\right)}\right| t\right) \text {. }
$$

Here $2 \check{n}_{3} \rho_{\left(0,0,2 n_{3}\right)}$ is vertical shearing of horizontal spatial average of $\rho$ which is an adiabatic invariant (time independent) in the asymptotic limit.

The term containing vertical shearing of $\bar{\rho}_{k}$ in $(3.17)$ is associated with the coefficient $\hat{Q}_{\bar{k} m n}^{012}$ given by (2.32). It involves the vertical shearing operator $\partial / \partial x_{3}$ (multiplication by $k_{3}$ in Fourier space). In this respect Eqs. (3.17) should be useful in detailed investigations of vertical mixing in times of high shear. Doppler effects such as shifting the frequency of an internal wave can be measured experimentally through Eqs. (3.21). We have shown the existence of a statistical dephasing effect, induced by turbulent processes on inertial-gravity waves. Turbulence acts to renormalize both frequency and viscosity of waves (Legras, 1980; Carnevale and Martin, 1982). Legras (1980) has investigated turbulent phase shifts of Rossby waves coupled with QG turbulence. Using EDQNM theory and numerical simulations he showed that in the turbulent domain turbulent frequency shifts can be as large as $30 \%$ of the wave frequency given by linear theory. We effectively generalize this work to the most general interactions of inertio-gravity waves with 3DQG turbulence. In BMN (1996a) we presented formulas for frequency shifts induced by turbulence on inertial waves in the context of $3 \mathrm{D}$ rotating Euler equations. The turbulent shifts for rotating shallow-water equations with the $\beta$ term were analyzed in BMN (1996 c,e).

The equations (3.17) for $\mathbf{w}_{\boldsymbol{A} G}$ are also invariant under vertical averaging, $k_{3}=m_{3}=n_{3}=0$; this follows from the equivalent condition for two wave resonances, $\omega_{m}=\omega_{n}$ implies $\left|m_{3}\right| /|m|=\left|n_{3}\right| /|n|$. Denote by $\overline{\mathbf{w}}_{A G}$ the vertical average of $\mathbf{w}_{A G}$. Careful inspection of the coefficients $\hat{Q}_{k m n}^{i_{1} i_{2} i_{3}}$ in (3.16) shows that they all reduce to zero, except for:

$$
\hat{Q}_{\overline{\bar{k}} \overline{\bar{m}} \overline{\bar{n}}}^{011}=\hat{Q}_{\bar{k} \overline{\bar{m}} \bar{n}}^{022}=\frac{\check{k}^{\prime} \wedge \check{m}^{\prime}}{|\dot{k}|}
$$

Moreover the Craya-Herring basis reduces to $q_{0 \overline{\bar{n}}}=p_{0 \overline{\bar{n}}}, q_{1 \overline{\bar{n}}}=(0,0,-1,0), q_{2} \overline{\bar{n}}=(0,0,0,1)$. Hence $\overline{\overline{\mathbf{w}}}_{A G}$ reduces to the vertical averages of vertical velocity and buoyancy variables. Vertical averaging is usually denoted as the barotropic component (Bartello, 1995). The above establishes that these quantities are 
purely passively advected by the $2 \mathrm{D}-2 \mathrm{C}$ vertically averaged quasigeostrophic velocities. The linkage with the dynamical Taylor-Proudman theorem at $B u=0$ is remarkable, as $\overline{\bar{u}}_{3}$ and $\overline{\bar{\rho}}$ are indeed passively advected by the $2 \mathrm{D}-2 \mathrm{C}$ vertically averaged Euler fields in that context too. It confirms that barotropic components $\overline{\bar{u}}_{3}$ and $\overline{\bar{\rho}}$ of the field take no part in the geostrophic adjustment process.

Further, for almost all $B u$ and domain aspect ratios $a_{2}, \mathbf{B}_{2}\left(\mathbf{w}_{Q G}, \mathbf{w}_{A G}\right)$ in (3.17) splits in Fourier space into uncoupled, restricted interaction operators on 4 rays families

$$
\left(\begin{array}{l}
m_{1} \\
m_{2} \\
m_{3}
\end{array}\right)=\lambda\left(\begin{array}{ccc} 
\pm 1 & 0 & 0 \\
0 & \pm 1 & 0 \\
0 & 0 & \pm 1
\end{array}\right)\left(\begin{array}{l}
n_{1} \\
n_{2} \\
n_{3}
\end{array}\right)
$$

for $n_{3} \neq 0$. This is obtained by further reducing resonances $\omega_{m}=\omega_{n}$. In Eqs. (3.17) together with the condition (3.23) direct cascades of energy are allowed for $\mathbf{w}_{A G}$ through $\mathbf{B}_{2}\left(\mathbf{w}_{Q G}, \mathbf{w}_{A G}\right)$. Wave energy cascades toward smaller scales and is subject to strong dissipation in AG mode. Vertical shearing operations in $\mathbf{B}_{2}\left(\mathbf{w}_{Q G}, \mathbf{w}_{A G}\right)$ are conveniently expressed in terms of divergent velocity potential $\chi$. We recall that $\chi$ and $\psi$ are related to $\mathbf{U}_{h}$ by the formula $\mathbf{U}_{h}=e_{3} \times \nabla_{h} \psi+\nabla_{h} \chi$. The form $\mathbf{B}_{2}\left(\mathbf{w}_{Q G}, \mathbf{w}_{A G}\right)$ takes an especially simple form in the Craya-Herring cyclic basis. In this basis the first component of $\mathbf{w}_{A G}$ is simply the divergent velocity potential $\chi=(-\Delta)_{h}^{-1} \frac{\partial U_{A G}^{3}}{\partial x_{3}}$ or in Fourier space

$$
\chi_{n}=-i \frac{\check{n}_{3}}{|\check{n}|} \frac{1}{\left|\check{n}^{\prime}\right|} w_{n}^{1}
$$

where $\check{n}^{\prime}=\left(n_{1}, n_{2} / a_{2}\right)$, and the components of the full field in the Craya-Herring basis are $\left(w_{n}^{0}, w_{n}^{1}, w_{n}^{2}\right)$. Here $w_{n}^{0}$ is the component of the quasi-geostrophic mode and $w_{n}^{1}, w_{n}^{2}$ are the ageostrophic components.

The coefficients $\tilde{Q}_{k m n}^{011}=\tilde{Q}_{k m n}^{011}, \tilde{Q}_{k m n}^{012}, \tilde{Q}_{k m n}^{021}$ in (3.15)-(3.16) explicitly yield terms $\chi_{m}=-i \frac{\check{m}_{3}}{\left|\check{m}^{\prime}\right|} \frac{1}{\left|\check{m}^{\prime}\right|} w_{m}^{1}$, $\chi_{n}=-i \frac{\check{n}_{3}}{|\tilde{n}|} \frac{1}{\left|\tilde{n}^{\prime}\right|} w_{n}^{1}$ which reflect vertical shearing dynamics, and contribute to imbalance dynamics. These terms come from $Q_{k m n}^{011}, Q_{k m n}^{012}, Q_{k m n}^{021}, Q_{m k n}^{201}, Q_{m k n}^{101}$ given by Eqs. (2.25)-(2.27) and are carried over to the $\hat{Q}_{k m n}^{011}, \hat{Q}_{k m n}^{012}, \hat{Q}_{k m n}^{021}$ in Eqs. (2.31) and in Eqs. (3.15)-(3.16). The important role of the divergent velocity potential $\chi$ in the AG reduced Eqs. (3.17) can be compared with that in the classical balanced models (Gent and McWilliams, 1983a, b). There a classical expansion in small parameters is carried. In some sense, our QG+AG Eqs. (3.17) describe near balanced and some unbalanced regimes.

Wave energy cascades towards smaller scales along rays in Fourier space and is subject to strong dissipation in the ageostrophic mode. We have an upscale energy cascade via $(0,0,0)$ triads corresponding to 3DQG, downscale cascade of energy via $\left(0, i_{2}, i_{3}\right), i_{2} \neq 0, i_{3} \neq 0$ triads corresponding to catalytic interactions. Triads $\left(0, i_{2}, i_{3}\right), i_{2} \neq 0, i_{3} \neq 0$ flush the gravity wave energy downscale in a nonlinear geostrophic adjustment process. When simulations are initially balanced, gravity modes act to damp large scale rotational modes via transfer into intermediate scale gravity modes, and via a subsequent downscale wave cascade involving 
the catalytic interaction. Effective eddy viscosity on fast modes is larger than that on slow modes confirming Bartello et al. (1996). Spectral eddy viscosities for QG and AG fields with explicit dependence on the rotation/stratification parameters are calculated. They correspond to 2 -wave resonances on the linear diffusion operator. Let $\nu_{1}$ and $\nu_{2}$ be the kinematic viscosity and the heat conductivity, respectively; the ratio $\nu_{1} / \nu_{2}$ is known as the Prandtl number. Then effective spectral eddy viscosities $\nu_{Q G}$ and $\nu_{A G}$ are given by

$$
\nu_{Q G}(n)=\nu_{2}+\left(\nu_{1}-\nu_{2}\right) \frac{\left|\check{n}^{\prime}\right|^{2}}{\left|\check{n}^{\prime}\right|^{2}+\mu^{2} \breve{n}_{3}^{2}}, \nu_{A G}(n)=\nu_{1}+\left(\nu_{2}-\nu_{1}\right) \frac{\left|\check{n}^{\prime}\right|^{2}}{\left|\check{n}^{\prime}\right|^{2}+\mu^{2} \check{n}_{3}^{2}}
$$

where $\mu=f / N$. Let's consider the case of a large Prandtl number $\nu_{1} / \nu_{2}>>1$. Then Eqs. (3.25) can be approximated by the following expressions

$$
\nu_{Q G}(n)=\nu_{1} \frac{\left|\check{n}^{\prime}\right|^{2}}{\left|\check{n}^{\prime}\right|^{2}+\mu^{2} \check{n}_{3}^{2}}, \nu_{A G}(n)=\nu_{1} \frac{\mu^{2} \check{n}_{3}^{2}}{\left|\check{n}^{\prime}\right|^{2}+\mu^{2} \check{n}_{3}^{2}} .
$$

Eqs. (3.26) clearly show that QG and AG modes are effected by viscosity differently. For example, $\nu_{A G}(n$ ) increases if vertical shearing increases (large $n_{3}$ ) while $\nu_{Q G}(n)$ decreases. The dependence of effective eddy viscosities on $\mu=f / N$ is also of interest. In the stratification dominated regime $(\mu \rightarrow 0)$ we have $\nu_{Q G}(n) \rightarrow$ $\nu_{1}, \nu_{A G}(n) \rightarrow 0$. The fact that $\nu_{A G}(n) \rightarrow 0$ is reflected in lack of control of AG dynamics (vertical shearing) in the strongly stratified limit. The situation changes dramatically in the rotation dominated regime $\mu \rightarrow+\infty$. In this situation we have $\nu_{A G}(n) \rightarrow \nu_{1}, \nu_{Q G}(n) \rightarrow 0$. The fact that $\nu_{A G}(n)$ approaches a nonzero finite value allows us to control AG dynamics in this limit. Remarkably, $\nu_{Q G}(n) \rightarrow 0$ does not spoil the situation since QG dynamics is globally controlled even for zero viscosity (Bourgeois and Beale, 1994). In the physical case $\operatorname{Pr}=\nu_{1} / \nu_{2} \neq 1$ and naively adding the usual viscosity to 3DQG equations is incorrect in the geophysical limit.

As noticed by Métais and Herring (1987) and Métais et al. (1996), the presence of stratification yields the formation of very strong vertical variability which tends to destroy the vortices vertical coherence. In stably stratified, rotating flows, quasi-two-dimensional organized vortices structures are only observed in the presence of a well-defined horizontal density (temperature) front leading to baroclinic instability.

The impact of even small viscosity onto ageostrophic dynamics in (3.17) is strikingly different from that on the quasi-geostrophic equations (3.12) confirming the predictions of Métais and Herring (1989). In (3.12) modified with $\nu_{Q G}$ dissipative small scales interact nonlinearly with an anisotropic inertial range with inverse cascades and intermittencies in the quasi-geostrophic turbulence. Inviscid ageostrophic dynamics are driven by the chaotic quasi-geostrophic field, albeit on restricted rays in the Fourier wavenumber space (but no restriction to the direct energy cascade). Energy is conserved separately on each family, but not enstrophy. Direct cascade of energy is enhanced along each ray family, in contrast to the inverse cascade for the QG component spectrum. This is confirmed by numerical observations of Métais et al. (1996). 


\section{Intermediate asymptotic regime of strong stratification and weak rotation}

In this section only $k_{2} / a_{2}$ will be denoted by $k_{2}$ and $k_{3} / a_{3}$ by $k_{3},|k|^{2}=k_{1}^{2}+k_{2}^{2} / a_{2}^{2}+k_{3}^{2} / a_{3}^{2},\left|k^{\prime}\right|^{2}=k_{1}^{2}+k_{2}^{2} / a_{2}^{2}$. Intermediate asymptotic regime of strong stratification and weak rotation is conveniently characterized in the Craya-Herring cyclic basis using expansions in a small parameter $\mu=f / N=f_{0} / N_{0}$. Equations describing balanced dynamics with control of vertical shearing are obtained by expanding $Q_{k m n}^{000}=\frac{N \omega_{m}|m|}{\omega_{k}|k| \omega_{n}|n|}\left(k^{\prime} \wedge m^{\prime}\right)$ in Eqs. (2.24) in powers of $\mu$. We have

$$
\begin{array}{r}
\omega_{k}|k|=\sqrt{N^{2}\left|k^{\prime}\right|^{2}+f^{2} k_{3}^{2}}=N \sqrt{\left|k^{\prime}\right|^{2}+\mu^{2} k_{3}^{2}}=N\left|k^{\prime}\right|\left(1+\frac{k_{3}^{2}}{2\left|k^{\prime}\right|^{2}} \mu^{2}+O\left(\mu^{4}\right)\right), \\
\frac{N}{\omega_{k}|k|}=\frac{1}{\sqrt{\left|k^{\prime}\right|^{2}+\mu^{2} k_{3}^{2}}}=\frac{1}{\left|k^{\prime}\right|}\left(1-\frac{k_{3}^{2}}{2\left|k^{\prime}\right|^{2}} \mu^{2}+O\left(\mu^{4}\right)\right)
\end{array}
$$

with similar expressions for $\omega_{m}|m|$ and $\omega_{n}|n|$. Here $O\left(\mu^{4}\right)$ denote terms in Taylor series of order $\mu^{4}$ and

higher. Expansions (4.1) are valid provided that $\frac{k_{3}^{2}}{\left|k^{\prime}\right|^{2}} \mu^{2}<<1$. We note that $l_{v}=1 /\left|k_{3}\right|$ and $l_{h}=1 /\left|k^{\prime}\right|$ are the vertical and horizontal spectral length scales, respectively. Then the condition $\frac{k_{3}^{2}}{\left|k^{\prime}\right|^{2}} \mu^{2}<<1$ is equivalent to the condition $B u_{s} \equiv N_{0}^{2} l_{v}^{2} / f_{0}^{2} l_{h}^{2}>>1$. Here $B u_{s}$ is the spectral Burger number.

Substituting expansions (4.1) in the expression for $Q_{k m n}^{000}$ we obtain after cancelling $N^{2}$ and suppressing terms of order $\mu^{4}$ and higher

$$
Q_{k m n}^{000}(\mu)=\frac{\left|m^{\prime}\right|}{\left|k^{\prime}\right|\left|n^{\prime}\right|}\left(k^{\prime} \wedge n^{\prime}\right)\left[1+\frac{1}{2} \mu^{2}\left(\frac{m_{3}^{2}}{\left|m^{\prime}\right|^{2}}-\frac{k_{3}^{2}}{\left|k^{\prime}\right|^{2}}-\frac{n_{3}^{2}}{\left|n^{\prime}\right|^{2}}\right)\right]+O\left(\mu^{4}\right) .
$$

Clearly,

$$
Q_{k m n}^{000}(0)=\frac{\left|m^{\prime}\right|}{\left|k^{\prime}\right|\left|n^{\prime}\right|}\left(k^{\prime} \wedge n^{\prime}\right)
$$

The appearance of the operator $k_{3}^{2} /\left|k^{\prime}\right|^{2}$ etc. in Eqs. (4.3) is not surprising since it is related to the inversion of (3.10). Indeed, for small $\mu$ we have

$$
\tilde{\psi}=-\left(\nabla_{h}^{2}+\mu^{2} \frac{\partial^{2}}{\partial x_{3}^{2}}\right)^{-1} \tilde{q}=-\nabla_{h}^{-2}\left(1-\mu^{2} \nabla_{h}^{-2} \frac{\partial^{2}}{\partial x_{3}^{2}}\right)+O\left(\mu^{4}\right)
$$

where the operator $-\nabla_{h}^{-2} \frac{\partial^{2}}{\partial x_{3}^{2}}$ is $-k_{3}^{2} /\left|k^{\prime}\right|^{2}$ in Fourier space.

Eqs. (4.3) show that the regularizing effect of rotation involves the inverses of horizontal Laplacian. The effect of these operators on the dynamics is to decrease the horizontal scale. This is in fact what is observed in numerical simulations investigating the effects of rotation on turbulence (McWilliams, private communication). 
Eqs. (3.7) now take the form

$$
\partial_{t} w_{n}^{0}=-i \sum_{k+m=n} Q_{k m n}^{000}(\mu) w_{k}^{0} w_{m}^{0}
$$

where $Q_{k m n}^{000}(\mu)$ is given by Eqs. (4.3). For every $\mu \neq 0$ the coefficients $Q_{k m n}^{000}(\mu)$ have explicit dependence on vertical variability parameters $\left(k_{3}, m_{3}, n_{3}\right.$ in Fourier space). For $\mu=0$ dependence on vertical variable disappears and we obtain complete decoupling of dynamics at every vertical layer with the vertical coordinate $x_{3}$ appearing only as a parameter. At any given vertical level the dynamics is described by $2 \mathrm{D}$ Euler equations with different pressures at every vertical level. There is no control of vertical shearing which can grow exponentially. We obtain quasi-2D unbalanced dynamics of vertically stacked pancakes which was first described by Lilly (1983). For $\mu \neq 0$, as shown below, Eqs. (4.3), (4.6) have a conservation law which allows to control vertical shearing for all times. Our results reveal the regularizing effect of weak rotation which introduces vertical coherence; the mechanism of the regularization is through coupling of dynamics in the vertical direction. This regularization differs from regularizations based on vertical viscosity.

We note that the coefficients $Q_{k m n}^{000}(\mu)$ have the following important skew-symmetric property

$$
Q_{k m n}^{000}(\mu)=-Q_{n m k}^{00 \mathrm{C}}(\mu)
$$

Eqs. (4.6) have a conservation law which simply expresses the fact that potential vorticity is conserved if written in $\tilde{q}$ variables. Multiplying (4.6) by $w_{-n}^{0}$ and summing over $n$ we obtain

$$
\partial_{t} \sum_{n}\left|w_{n}^{0}(t)\right|^{2}=0
$$

since

$$
-i \sum_{k+m=n} Q_{k m n}^{000}(\mu) w_{k}^{0} w_{m}^{0} w_{-n}^{0}=-i \sum_{k+m+n=0} Q_{k m n}^{000}(\mu) w_{k}^{0} w_{m}^{0} w_{n}^{0}=0 .
$$

In (4.9) we used the fact that $Q_{k m n}^{000}(\mu)$ is anti-symmetric (Eqs. (4.7)) and $w_{k}^{0} w_{m}^{0} w_{n}^{0}$ is symmetric under interchange of $k$ and $n$. The conservation law for $w_{n}^{0}(t)$ implies conservation of energy for the projection of the field on

$$
q_{o n}(\mu)=\left(1-\frac{n_{3}^{2}}{2\left|n^{\prime}\right|^{2}} \mu^{2}\right) p_{o n}+\mu \frac{n_{3}}{\left|n^{\prime}\right|} p_{2 n}+O\left(\mu^{3}\right) .
$$

Then using the conservation law (4.8) and following Bourgeois and Beale (1994) one can prove global existence of solutions of Eqs. (4.3), (4.6) with full control of vertical shearing for all times.

The coefficients $Q_{k m n}^{i_{1} i_{2} i_{3}}(\mu)$ given by Eqs. (2.25)-(2.28) can be expanded in powers of $\mu$ (see Appendix). Then we obtain

$$
Q_{k m n}^{000}(\mu)=Q_{k m n}^{000}(0)+\frac{1}{2} \mu^{2} \frac{\left|m^{\prime}\right|}{\left|k^{\prime}\right|\left|n^{\prime}\right|}\left(k^{\prime} \wedge n^{\prime}\right)\left(\frac{m_{3}^{2}}{\left|m^{\prime}\right|^{2}}-\frac{k_{3}^{2}}{\left|k^{\prime}\right|^{2}}-\frac{n_{3}^{2}}{\left|n^{\prime}\right|^{2}}\right)+O\left(\mu^{4}\right),
$$




$$
\begin{gathered}
\hat{Q}_{k m n}^{011}(\mu)=\hat{Q}_{k m n}^{011}(0)-\frac{1}{2} \mu^{2} \frac{k_{3}^{2}\left(k^{\prime} \wedge m^{\prime}\right)\left(n_{3} m_{3} n^{\prime} \cdot m^{\prime}+\left|m^{\prime}\right|^{2}\left|n^{\prime}\right|^{2}+n_{3} m_{3} k^{\prime} \cdot m^{\prime}-n_{3} k_{3}\left|m^{\prime}\right|^{2}\right)}{\left|k^{\prime}\right|^{3}\left|m^{\prime}\right|\left|n^{\prime}\right||m||n|} \\
\hat{Q}_{k m n}^{022}(\mu)=\hat{Q}_{k m n}^{022}(0)-\frac{1}{2} \mu^{2} \frac{k^{\prime} \wedge m^{\prime}}{\left|k^{\prime}\right|}\left(\frac{k_{3}^{2}}{\left|k^{\prime}\right|^{2}}+\frac{m_{3}^{2}}{\left|m^{\prime}\right|^{2}}+\frac{n_{3}^{2}}{\left|n^{\prime}\right|^{2}}\right)+ \\
\mu^{2} \frac{\left(k^{\prime} \wedge m^{\prime}\right)\left(m_{3} n_{3} m^{\prime} \cdot n^{\prime}+m_{3} k_{3}\left|n^{\prime}\right|^{2}-m_{3} n_{3} n^{\prime} \cdot k^{\prime}\right)}{\left|k^{\prime}\right|\left|m^{\prime}\right|\left|n^{\prime}\right|\left|m^{\prime}\right|\left|n^{\prime}\right|}+O\left(\mu^{4}\right) \\
\hat{Q}_{k m n}^{012}(\mu)=-2 \mu \frac{m_{3} n_{3}\left(k^{\prime} \wedge m^{\prime}\right)^{2}+\left(m_{3} k^{\prime} \cdot m^{\prime}-k_{3}\left|m^{\prime}\right|^{2}\right)\left(n_{3} n^{\prime} \cdot k^{\prime}-k_{3}\left|n^{\prime}\right|^{2}\right)}{\left|k^{\prime}\right|\left|n^{\prime}\right|\left|m^{\prime}\right|\left|m^{\prime}\right|\left|n^{\prime}\right|}+O\left(\mu^{3}\right), \\
\hat{Q}_{k m n}^{021}(\mu)=4 \mu \frac{m_{3} n_{3}\left(k^{\prime} \wedge m^{\prime}\right)^{2}}{|n|\left|m^{\prime}\right|\left|k^{\prime}\right|\left|m^{\prime}\right|\left|n^{\prime}\right|}+O\left(\mu^{3}\right)
\end{gathered}
$$

where

$$
\begin{aligned}
& Q_{k m n}^{000}(0)=\frac{\left|m^{\prime}\right|}{\left|k^{\prime}\right|\left|n^{\prime}\right|}\left(k^{\prime} \wedge n^{\prime}\right), \hat{Q}_{k m n}^{022}(0)=\frac{k^{\prime} \wedge m^{\prime}}{\left|k^{\prime}\right|}, \hat{Q}_{k m n}^{012}(0)=\hat{Q}_{k m n}^{021}(0)=0 \\
& \hat{Q}_{k m n}^{011}(0)=\frac{\left(k^{\prime} \wedge m^{\prime}\right)\left(n_{3} m_{3} n^{\prime} \cdot m^{\prime}+\left|m^{\prime}\right|^{2}\left|n^{\prime}\right|^{2}+n_{3} m_{3} k^{\prime} \cdot m^{\prime}-n_{3} k_{3}\left|m^{\prime}\right|^{2}\right)}{\left|k^{\prime}\right|\left|m^{\prime}\right|\left|n^{\prime}\right||m||n|}
\end{aligned}
$$

Our regularized system for $\mathbf{w}_{n}=\left(w_{n}^{0}, w_{n}^{\prime}\right), \mathbf{w}_{n}^{\prime}=\left(w_{n}^{1}, w_{n}^{2}\right)$ follows from Eqs. (3.7), (3.16)-(3.17). It has the form

$$
\begin{gathered}
\partial_{t} w_{n}^{0}=-i \sum_{k+m=n} Q_{k m n}^{000}(\mu) w_{k}^{0} w_{m}^{0}+O\left(\mu^{4}\right), \\
\partial_{t} \mathbf{w}_{n}^{\prime}=i \sum_{\substack{\phi_{m}=\phi_{n} \\
k+m=n}} \frac{N \phi_{m} \phi_{n}}{\omega_{n}} k_{3 \tilde{\rho}_{k}} \mathbf{J}_{\mathbf{w}_{m}^{\prime}-i} \sum_{\substack{\phi_{m}=\phi_{n} \\
k+m=n}} w_{k}^{0}(t) \tilde{\mathbf{Q}}_{k m n}(\mu) \mathbf{w}_{m}^{\prime}+O\left(\mu^{4}\right) .
\end{gathered}
$$

where

$$
\begin{gathered}
\tilde{\mathbf{Q}}_{k m n}(\mu)=\left(\begin{array}{cc}
D_{k m n}(\mu), & G_{k m n}(\mu) \\
-G_{k m n}(\mu), & D_{k m n}(\mu)
\end{array}\right)=D_{k m n}(\mu)\left(\begin{array}{ll}
1 & 0 \\
0 & 1
\end{array}\right)+G_{k m n}(\mu)\left(\begin{array}{cc}
0 & 1 \\
-1 & 0
\end{array}\right) \\
D_{k m n}(\mu)=\hat{Q}_{k m m n}^{0 \vdots 1}(\mu)+\hat{Q}_{k m n}^{022}(\mu), G_{k m n}(\mu)=-\hat{Q}_{k m n}^{012}(\mu)+\hat{Q}_{k m n}^{021}(\mu)
\end{gathered}
$$

with components of the matrix $\tilde{\mathbf{Q}}_{k m n}(\mu)$ given by formulas above. For every fixed value of the parameter $\mu$ vertical shearing is controlled in the reduced equations (4.18)-(4.20) for all times. The system of equations (4.18)-(4.20) will be called the regularized Boussinesq system in the regime of strong stratification and weak 
rotation. It allows us to study the regularizing effect of weak rotation on quasi-2D dynamics of decoupled pancakes and its impact on AG dynamics. For $\mu=0$ we obtain

$$
G_{k m n}(0)=\hat{Q}_{k m n}^{021}(0)-\hat{Q}_{k m n}^{012}(0)=0, D_{k m n}(0)=\hat{Q}_{k m n}^{011}(0)+\hat{Q}_{k m n}^{022}(0)
$$

where $\hat{Q}_{k m n}^{011}(0)$ and $\hat{Q}_{k m n}^{022}(0)$ are given in $(4.16)-(4.17)$. We recall that $w_{n}^{1}$ and $w_{n}^{2}$ are projections of the total field on the divergent velocity potential $(\chi)$ and the geostrophic departure mode, respectively. The matrix $\tilde{\mathbf{Q}}_{k m n}(0)$ is diagonal and equations (4.19) for $w_{n}^{1}$ and $w_{n}^{2}$ are coupled for $\mu=0$ only through the phase term $i \frac{N \phi_{m} \phi_{n}}{\omega_{n}} k_{3} \bar{\rho}_{k} \mathbf{J}_{\mathbf{w}_{m}}^{\prime}$ which is associated with vertical shearing of horizontally averaged buoyancy variable (adiabatic invariant). For $\mu \neq 0$ the modes $w_{n}^{1}$ and $w_{n}^{2}$ are coupled as can be seen from Eqs. (4.20). Non-diagonal terms are proportional to $G_{k m n}(\mu)$; Taylor series expansion of $G_{k m n}(\mu)$ starts with terms of order $\mu$.

In order to obtain a simple reduced system suitable for practical numerical implementation, terms of order $\mu$ in Eqs. (4.19) are neglected. Then two scalar equations for $w^{1}$ and $w^{2}$ in (4.19) are coupled only through the phase term and can be solved in parallel.

A simplified reduced system consists of three prognostic equations:

$$
\begin{gathered}
\partial_{t} w_{n}^{0}=-i \sum_{k+m=n} Q_{k m n}^{000}(\mu) w_{k}^{0} w_{m}^{0}, \\
\partial_{t} \mathbf{w}_{n}^{\prime}=i \sum_{\substack{\phi_{m}=\phi_{n} \\
k+m=n}} \frac{N \phi_{m} \phi_{n}}{w_{n}} k_{3} \bar{\rho}_{k} \mathbf{J} \mathbf{w}_{m}^{\prime}-i \sum_{\substack{\phi_{m}=\phi_{n} \\
k+m=n}} w_{k}^{0}(t) D_{k m n}(0) \mathbf{w}_{m}^{\prime} .
\end{gathered}
$$

The scalar coefficient $D_{k m n}(0)$ in (4.24) is given by

$$
D_{k m n}(0)=\hat{Q}_{k m n}^{011}(0)+\hat{Q}_{k m n}^{022}(0)=2 \frac{k^{\prime} \wedge m^{\prime}}{\left|k^{\prime}\right|} \frac{n_{3} m_{3} n^{\prime} \cdot m^{\prime}+\left|m^{\prime}\right|^{2}\left|n^{\prime}\right|^{2}}{\left|m^{\prime}\right|\left|n^{\prime}\right||m||n|}
$$

In the derivation of (4.25) we used the relation $\phi_{m}=\phi_{n}$. The first term $\frac{k^{\prime} \wedge m^{\prime}}{\left|k^{\prime}\right|}$ in (4.25) represents advection in physical space. Now we analyze the role of the second 0 -order term

$$
\gamma(n, m)=\frac{n_{3} m_{3} n^{\prime} \cdot m^{\prime}+\left|m^{\prime}\right|^{2}\left|n^{\prime}\right|^{2}}{\left|m^{\prime}\right|\left|n^{\prime}\right||m||n|}=\frac{\left|n^{\prime} \wedge m^{\prime}\right|^{2}+(n \cdot m)\left(n^{\prime} \cdot m^{\prime}\right)}{\left|m^{\prime}\right|\left|n^{\prime}\right||m||n|} .
$$

The coefficient $\gamma(n, m)$ can be easily computed for 4 ray families. For example, we obtain from (4.26)

$$
\begin{array}{r}
\gamma(n, m)=1 \text { if } m_{1}=\lambda n_{1}, m_{2}=\lambda n_{2}, m_{3}=\lambda n_{3} \\
\gamma(n, m)=\frac{m_{1}^{2}+m_{2}^{2}-m_{3}^{2}}{|m|^{2}} \text { if } m_{1}=\lambda n_{1}, m_{2}=\lambda n_{2}, m_{3}=-\lambda n_{3} .
\end{array}
$$


The appearance of a wave type operator $\frac{m_{1}^{2}+m_{2}^{2}-m_{3}^{2}}{|m|^{2}}$ in Eqs. (4.24) is due to polarization induced by waves; it corresponds to wave dynamics on the reduced family of 4 rays.

The advantage of prognostic equations (4.23)-(4.24) over the diagnostic vertical velocity (omega) equation (1.17) is in improved accuracy. Near balance and some unbalanced regimes can be treated by Eqs.(4.23)(4.24). These regimes cannot be treated by the diagnostic equation (1.17) where filtered initial data is required in order for (1.17) to be a reasonable approximation of (1.16).

For $\mu=0$ Eqs. (4.18) coincide with the familiar quasi-2D Euler system which can be seen by introducing variables $\tilde{q}$ and $\mathbf{U}_{Q 2 D}$ (quasi2D potential and velocity):

$$
\tilde{q}_{m}=\left|m^{\prime}\right| w_{m}^{0}, \quad \mathbf{U}_{Q 2 D, k}=\left[-k_{2}, k_{1}, 0,0\right] \tilde{q}_{k} /\left|k^{\prime}\right|^{2} .
$$

In this notation (3.7) is written in the form of $2 \mathrm{D}$ Euler equation which depends on $x_{3}$ as on a parameter:

$$
\partial_{t} \tilde{q}_{n}=-i \sum_{k+m=n}\left(\mathbf{U}_{Q 2 D, k} \cdot m\right) \tilde{q}_{m}
$$

Then the velocity $\mathbf{U}_{Q 2 D}\left(t, x_{1}, x_{2}, x_{3}\right)$ satisfies quasi-2D Euler system

$$
\partial_{t} \mathbf{U}_{Q 2 D}+\left(\overline{\mathbf{U}}_{Q 2 D}+\mathbf{U}_{Q 2 D}\right) \cdot \nabla^{\prime} \mathbf{U}_{Q 2 D}=-\nabla^{\prime} \tilde{p}, \nabla^{\prime} \cdot \mathbf{U}_{Q 2 D}=0
$$

which depend on $x_{3}$ as a parameter, $\nabla^{\prime}=\left[\partial_{1}, \partial_{2}\right]$. In Eqs. (4.31) $\overline{\mathbf{U}}_{Q 2 D}$ denotes horizontally averaged velocity which is an adiabatic invariant of $3 \mathrm{D}$ Boussinesq equations in the strongly stratified limit (in the absence of rotation).

\section{Phenomenological analysis at asymptotic limit of strong rota- tion/stratification in the Burger number of order one situation}

At asymptotic limit of strong rotation/stratification, the existence of two disparate time scales indicates a phenomenological analysis similar to that of rotation (Zhou, 1995; Mahalov and Zhou, 1996) may be appropriate. The aim of this approach is to estimate the averaged effect of rotation/stratification on turbulent energy transfer. The introduction of the anisotropic time scale based on the aspect ratio parameter in the energy spectrum is an improvement over our previous phenomenological analysis of rotating turbulence. In the context of the quasi-geostrophic equations for a Boussinesq fluid in a uniformly rotating and stably stratified environment, McWilliams et al. (1994) showed that their solutions exhibit significant anisotropy associated with the emergence of many long lived coherent vortices that control the flow evolution. Anisotropy of quasi-geostrophic field impacts on the ageostrophic gravity wave field through Eqs. (3.17). 
Among more mundane immediate consequences, exact operator splitting of the reduced equations (3.3) reach the very roots of the mechanisms of wave-vortex interactions. Let us designate by \langle\rangle the ensemble averaging for any field $\mathbf{U}^{\dagger}$, and by $\mathbf{U}_{F}^{\dagger}=\mathbf{U}^{\dagger}-\left\langle\mathbf{U}^{\dagger}\right\rangle$ the fluctuations. Then the Reynolds stress operator becomes:

$$
\left\langle\mathbf{W}_{Q G, F}, \mathbf{W}_{Q G, F}\right\rangle+\left\langle\mathbf{W}_{A G, F}, \mathbf{W}_{A G, F}\right\rangle+2\left\langle\mathbf{W}_{Q G, F}, \mathbf{W}_{A G, F}\right\rangle
$$

The last tensor we shall designate as the anisotropic phase coherence tensor. It correlates the fluctuations of the ageostrophic $\mathbf{W}_{A G}$-field with the quasi-geostrophic $\mathbf{W}_{Q G}$-field. The anisotropic phase coherence tensor is the key player in the control of rapid 3-D pressure fluctuations. The dependence of the full Reynolds stress tensor on the intrinsic mean vorticity does not vanish in the limit of strongly rotating/stratified turbulence, as neither the field $\mathbf{W}_{A G}$, nor the "anisotropic phase coherence tensor" vanish. Long-lived phase coherence is an important part of turbulence (Bartello and Holloway, 1991; Herring and McWilliams, 1985).

In order to infer the form of the inertial-range spectrum, it is necessary to estimate the magnitude for the triple correlations. In general, $\tau_{3}$, the time scale for decay of triple correlations which is responsible for inducing turbulent spectral transfer, may depend on any relevant turbulence parameters. Because energy is conserved by the nonlinear interaction and a local cascade has been assumed, $\epsilon$ is independent of $k$. Local cascade also implies that $\epsilon$ is explicitly proportional to $\tau_{3}$ and depends on the wavenumber and on the power of the omni-directional energy spectrum. A simple dimensional analysis leads to

$$
\epsilon=A^{2} \tau_{3}(k) k^{4} E^{2}(k)
$$

where $A$ is a constant. We recall that $\omega_{k}=\sqrt{N_{0}^{2} \frac{\left|k^{\prime}\right|^{2}}{|k|^{2}}+f_{0}^{2} \frac{k_{3}^{2}}{|k|^{2}}}$. As in Section 4, we defined the vertical and the horizontal spectral scales as $l_{v}=\frac{1}{\left|k_{3}\right|}, l_{h}=\frac{1}{\left|k^{\prime}\right|}$. If $\eta=l_{v} / l_{h}=\left|k^{\prime}\right| /\left|k_{3}\right|$ is the ratio of these length scales then $\omega_{k}=\sqrt{N_{0}^{2} \frac{\eta^{2}}{\eta^{2}+1}+f_{0}^{2} \frac{1}{\eta^{2}+1}}$.

In the strongly rotating/stratified case when both effects are of the same order (Burger number of order one situation), the time scale for $\tau_{3}(k)$, the decorrelation of the triple velocity product, is the controlling parameter to influence the energy transfer process. In a regime of high Reynolds numbers and low Rossby and Froude numbers, turbulence is characterized by two disparate time-scales: a short anisotropic time scale associated with the rotation/stratification frequency $\tau_{\Omega N}=1 / \sqrt{N_{0}^{2} \frac{\eta^{2}}{\eta^{2}+1}+f_{0}^{2} \frac{1}{\eta^{2}+1}}\left(f_{0}=2 \Omega_{0}\right.$ is the Coriolis parameter) and a nonlinear time scale. We find that a direct application of $\tau_{3}=\tau_{\Omega N}$ resulted the energy spectrum for turbulence subject to strong rotation/stratification:

$$
E(k)=C_{\Omega N}\left(\tau_{\Omega N}^{-1} \epsilon\right)^{1 / 2} k^{-2}
$$

We now consider the number of the non-dimensional parameters needed. For the turbulence in equilibrium the Rossby/Froude numbers are the only relevant parameter controlling the effects of rotation/stratification 
on the flow. However, for the non-equilibrium situation, a new non-dimensional parameter such as $\left(\tau_{\Omega N}^{-1} t\right)$ is required. Here the dimensionality is considered by introducing the aspect ratio. Our analysis suggests that the energy transfer process in the limit of $\left(\tau_{\Omega N}^{-1} t\right) \rightarrow \infty$ and small spectral Rossby/Froude number (strong rotation/stratification) is as follows. There is inverse energy transfer by 3D QG component (McWilliams, Weiss and Yavneh, 1994). In the meantime, there is also a direct energy cascade governed by the equation for the inertio-gravity wave component. The energy spectrum of the full field $\mathbf{U}^{\dagger}$ is given by $E(k)=$ $C_{\Omega N}\left(\tau_{\Omega N}^{-1} \epsilon\right)^{1 / 2} k^{-2}$.

Following the usual assumption of EDQNM, we consider that the lifetime of triple correlations in rotating/stratified turbulence might be more accurately treated by taking into account the possibility that these correlations decay because of the influence of both wave propagation and nonlinear interactions. The simple choice

$$
\frac{1}{\tau_{3}(k)}=\frac{1}{\tau_{n l}(k)}+\frac{1}{\tau_{\Omega N}(k)}
$$

satisfies the appropriate limiting cases: $\tau_{3}(k) \rightarrow \tau_{n l}$ without rotation/stratification and $\tau_{3}(k) \rightarrow \tau_{\Omega N}$ with strong rotation/stratification. The introduction of the anisotropic time scale based on the aspect ratio parameter in the energy spectrum is an improvement over our previous phenomenological analysis (Zhou, 1995; Mahalov and Zhou, 1996) since now the model can distinguish the anisotropic nature of rotating/stratified flows. The generalized inertial range energy spectrum is

$$
E(k)=Z^{2} A^{-4 / 3} \epsilon^{2 / 3} k^{-5 / 3},
$$

where $Z$ is given by

$$
\begin{gathered}
Z=\frac{1}{2}\left(\sqrt{Y}+\sqrt{-Y+2 \sqrt{Y^{2}+4 Z_{0}}}\right) \\
Y=\sqrt[3]{\frac{1}{2}+\sqrt{\frac{1}{4}+\left(\frac{4 Z_{0}}{3}\right)^{3}}}+\sqrt[3]{\frac{1}{2}-\sqrt{\frac{1}{4}+\left(\frac{4 Z_{0}}{3}\right)^{3}}}
\end{gathered}
$$

The parameters $A=C_{K}^{-3 / 4}, k_{\Omega N}=\tau_{\Omega N}^{-3 / 2} \epsilon^{-1 / 2}$ and $Z_{0}=A^{2 / 3} \tau_{\Omega N}^{-1}\left(\epsilon k^{2}\right)^{-1 / 3}=\left[\frac{A k_{\Omega N}}{k}\right]^{2 / 3}$. The strong rotation/stratification limit then leads to $C_{\Omega N}=1 / A=1.22-1.87$ for the typical range of Kolmogorov constant. These equations reduce to the classical Kolmogorov "-5/3" spectrum when $Z_{0} \rightarrow 0$ (so that $Z \rightarrow 1$ ), and to our rotation/stratification modified "-2" spectrum when $Z_{0}>1$ (so that $Y \rightarrow 0, Z \rightarrow Z_{0}^{1 / 4}$ ). For intermediate rotation/stratification rates the spectrum varies smoothly between these two limiting forms, according to the increase of the controlling parameter $Z_{0}$ with increasing ratio $k_{\Omega N} / k$. This confirms the numerical simulations of rotating stratified turbulence by Métais et al. (1996). In their numerical simulations 
the geostrophic energy spectrum was steeper than $k^{-3}$ at the small scales, whereas the ageostrophic energy spectrum was much shallower. As a result, the large scales are dominated by geostrophic energy, while the reverse is true of the small scales. There is a spectral gap between QG and AG spectrum with AG spectrum being shallower than the typical $k^{-3} \mathrm{QG}$ spectrum; it varies smoothly between $k^{-2}$ and $k^{-5 / 3}$ which is in agreement with numerical simulations of Métais et al. (1996), Bartello et al. (1996).

The equation which determines the spectral eddy viscosity, $\nu_{T}(k)$, is given by

$$
\epsilon=\nu_{T}(k) S^{2}(k)=\nu_{T}(k) E(k) k^{3} .
$$

Therefore, the rotation/stratification dependent eddy viscosity can be estimated as

$$
\nu_{T}(k)=\nu_{0}\left[\frac{E(k)}{k}\right]^{1 / 2} \frac{1}{1+y^{-1 / 2}},
$$

where $\nu_{0}$ is a constant and $y=k^{3} E(k) / N^{2}$. The inertial range wavenumber $k$ can be related to the turbulent kinetic energy $K$ and dissipation rate $\epsilon$

$$
k=\frac{E(k)}{K^{3 / 2}}\left(3 C_{k} / 2\right)^{3 / 2} .
$$

For the inertial range spectrum, Eq. (5.9) can be rewritten in physical space:

$$
\nu_{T}(x)=\nu_{0}^{\prime} \frac{K^{2}}{\epsilon} \frac{1}{1+0.36 K /\left(\tau_{\Omega N} \epsilon\right)},
$$

The eddy viscosity above is only appropriate for homogeneous turbulence without mean velocity gradients.

The spectral time scale is an important measurement. The Kolmogorov hypothesis implies that the energy-containing range excitation does not affect energy transfer within the inertial range. Therefore, the average rate of energy dissipation is identified with the rate of spectral energy transfer and the rate of energy input. The nonlinear (or eddy turnover) time-scale, $\tau_{n l}(k) \equiv\left[k^{3} E(k)\right]^{-1 / 2}$, is then equivalent to the spectral transfer time, $\tau_{s}$. Zhou (1995) has shown that

$$
\tau_{s}(k)=\frac{1}{A^{2}} \frac{\left[\tau_{n l}(k)\right]^{2}}{\tau_{\Omega \Omega N}(k)},
$$

or more generally,

$$
\tau_{s}(k)=\frac{1}{A^{2}} \frac{\tau_{n l}^{2}(k)}{\tau_{3}(k)}
$$

Therefore, the time for the spectral energy transfer is increased to a value greater than $\tau_{n l}$; thus the nonlinear energy transfer is suppressed by rotation/stratification. 


\section{The devil's staircase of convergence results}

In this section we give a short presentation of new regularity and strong convergence results for rotating Boussinesq equations in the $B u=O(1)$ regime; these are substantial improvements over the corresponding ones in BMN (1996b) in that much less regularity is required for the initial data, with the Sobolev space $H_{9}$ being the worst and $H_{4}$ being the best case. Here $H_{\alpha}$ designates the space of functions with square integrable derivatives up to order $\alpha$. Any strong convergence result uniform in the parameters $R o_{a}$ or $1 / N$ cannot circumvent control of small divisors and sharp estimates of both near 3 - wave and near 2wave resonances. The $o(1)$ non-uniform convergence results in $H_{3}$ of Embid and Majda (1996) cannot be extended to $O(1 / N)$ uniform error without control of such near-resonances. Here we investigate the density and probability of both 3 -wave and 2-wave resonances as function of the three geometric parameters:

$$
B u=N^{2} a_{3}^{2} / f^{2}, \theta_{2}=1 / a_{2}^{2}, \theta_{3}=1 / a_{3}^{2}
$$

For rare, non-generic values of these parameters, the limit equations for the ageostrophic field $\mathbf{w}_{A G}$ can be nonlinear:

$$
\partial_{t} \mathbf{w}_{A G}=\mathbf{B}_{2}\left(\mathbf{w}_{Q G}(t), \mathbf{w}_{A G}\right)+\mathbf{B}_{3}\left(\mathbf{w}_{A G}, \mathbf{w}_{A G}\right)
$$

the bilinear form $\mathbf{B}_{3}$ can easily be computed in the Craya-Herring cyclic basis and is not detailed in this section for conciseness sake. In $\mathbf{B}_{3}$, the domain of summation $K^{*},(k, m) \in K^{*}, k+m=n$, depends on $\left(B u, 1 / a_{2}^{2}, 1 / a_{3}^{2}\right)=\left(B u, \theta_{2}, \theta_{3}\right)$; that is $K^{*}=K^{*}\left(B u, \theta_{2}, \theta_{3}\right)$. For every fixed $\theta_{2}=1 / a_{2}^{2}$ and $\theta_{3}=1 / a_{3}^{2}$, the summation set $K^{*}\left(B u, \theta_{2}, \theta_{3}\right)$ is not empty when $B u \in \Theta_{B}^{*}\left(\theta_{2}, \theta_{3}\right)$; the set $\Theta_{B}^{*}$ is very thin, namely it is countable. We call it a strict 3-wave resonant set. In Corollaries 6.1 and 6.2 we give an estimate of the very small probabilistic measure of near 3 -wave resonances. We mostly study the typical case when 3-wave interactions are absent, that is $B u \notin \Theta_{B}^{*}\left(\theta_{2}, \theta_{3}\right)$ and $\mathbf{B}_{3}\left(\mathbf{w}_{A G}, \mathbf{w}_{A G}\right)$ is identically zero. When $B u \in \Theta_{B}^{*}\left(\theta_{2}, \theta_{3}\right)$ is strictly resonant, $\mathbf{B}_{3}$ is non-zero and strongly depends on $B u$; the sets $K^{*}\left(B u, \theta_{2}, \theta_{3}\right)$ with different $B u$ do not intersect (highly nontrivial result from our study of the small divisor problem). This implies that the operator $\mathbf{B}_{3}$ depends on resonant $B u$ discontinuously, every point $B u \in \Theta_{B}^{*}\left(\theta_{2}, \theta_{3}\right)$ is a point of discontinuity of the operator $\mathbf{B}_{3}$. Since $\mathbf{B}_{3}$ is not zero, solutions of the limit system with general initial data discontinuously depend on $\mathrm{Bu}$ as well. As solutions of the original rotating Euler-Boussinesq equations depend on $B u$ continuously (on a small time interval $\left[0, T_{1}\right]$ ), the convergence to solutions of the limit equations cannot be uniform in $B u, a_{2}, a_{3}$. This paradox we call the "Devil's staircase of convergence results".

For $B u \notin \Theta_{B}^{*}$, Eqs. (6.2) reduces to the linear equations for catalytic interactions (3.16)-(3.17). At the same time, the equation strongly depends on $\theta_{2}=1 / a_{2}^{2}$, since the 2-wave resonance condition $\omega_{n}=\omega_{n}$ 
is equivalent to $\left|\check{m}^{\prime}\right| /\left|m_{3}\right|=\left|\check{n}^{\prime} /\right| n_{3} \mid$. For every irrational $\theta_{2}, \mathbf{B}_{2}$ splits in Fourier space into uncoupled, restricted interaction operators on 4 ray families as in (3.23). For every resonant rational point $\theta_{2} \in \Theta_{2}^{*}, \mathbf{B}_{2}$ includes more interactions (much larger, but finite number of Fourier rays), so indeed rational $\theta_{2}$ are also points of discontinuity for $\mathbf{B}_{2}$ and further contribute to the Devil's staircase of convergence. An important observation is that 2-wave resonances are controlled by $\theta_{2}$ only, not by $B u$ or $a_{3}$. This follows from the fact that $\omega_{m}=\omega_{n}$ implies $\left|m_{3}\right| /\left|\hat{m}^{\prime}\right|=\left|n_{3}\right| /\left|\hat{n}^{\prime}\right|$. In contrast, 3-wave resonances are controlled by Bu uniformly in $\theta_{2}$ and $\theta_{3}$; although strict resonant values $B u=\Theta_{B}^{*}\left(\theta_{2}, \theta_{3}\right)$ depend on $\theta_{2}$ and $\theta_{3}$, we prove that the estimate of the measure of almost resonant $B u$ does not depend on $\theta_{2}$ and is uniform in $\theta_{3}$. The sensitive dependence of convergence results on the parameters $B u$ and $a_{2}, a_{3}$ was missed by Embid and Majda. In fact:

\section{Lemma 6.1:}

Let $N^{2}=f^{2}$ (that is $\left.B u=a_{3}^{2}\right)$. Then there exist no 3 - wave resonances for $1 / 9 \leq a_{3}^{2} \leq 9$.

The main convergence result of this section (Theorem 6.1) shows that the convergence is uniform and the error is of order $\left(1 / \mu_{2}+1 / \mu_{3}\right) / N$ when $\theta_{2} \notin \Theta_{2}^{\mu_{2}}, B u \notin \Theta_{B}^{\mu_{3}}\left(\theta_{2}, \theta_{3}\right)$, with the Lebesgue measure meas $\left(\Theta_{B}^{\mu_{3}}\right) \leq \mu_{3}$, meas $\left(\Theta_{2}^{\mu_{2}}\right) \leq \mu_{2}$ with $\mu_{2}, \mu_{3}$ arbitrary small. $\Theta_{B}^{\mu_{3}}$ and $\Theta_{2}^{\mu_{2}}$ are the sets of near resonant 3 waves and 2 waves. Here $\|\cdot\|_{\alpha}$ designates the norm in the Sobolev space $H_{\alpha}$.

\section{Theorem 6.1:}

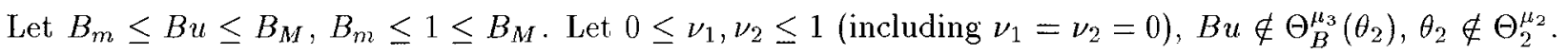
Let $\alpha>3 / 2, \sigma-\alpha>7, M_{0 \sigma}>0, \mu_{2}, \mu_{3} \leq 1$. Let $\left\|\mathbf{U}^{\dagger}(0)\right\|_{\sigma} \leq M_{0 \sigma}$. Let $\mathbf{U}^{\dagger}(t)$ be an exact solution of the $3 \mathrm{D}$ Euler-Boussinesq equations. Let $\mathbf{W}_{Q G}(t)$ be the solution to the QG equations (3.7), (3.11), (3.12) with initial data $\Pi^{Q G} \mathbf{U}^{\dagger}(0)$, and $\mathbf{w}_{A G}(t)$ the solution to the limit ageostrophic equations (3.17) on the 4 rays (3.23) with initial data $\Pi^{A G} \mathbf{U}^{\dagger}(0)$. Let $\mathbf{E}(t)$ be the inertio-gravity waves linear propagator. Then for $0 \leq t \leq T_{1}$

$$
\begin{aligned}
\left\|\mathbf{U}^{\dagger}(t)-\mathbf{W}_{Q G}(t)-\mathbf{E}(-t) \mathbf{w}_{A G}(t)\right\|_{\alpha} & \leq C a_{3}^{2}\left(1 / \mu_{2}+a_{3}^{2} / \mu_{3}\right) / N, \text { for } f<N, \\
& \leq C R o_{u} a_{3}\left(1 / \mu_{2}+a_{3}^{2} / \mu_{3}\right), \text { for } f>N
\end{aligned}
$$

where $T_{1}$ depends on only on $M_{0 \sigma} ; C$ depends only on $M_{0 \sigma}, \alpha, B_{M}$ (for $f<N$ ) or $B_{m}$ (for $f>N$ ).

\section{Theorem 6.2:}

For $\Pi^{Q G} \mathbf{U}^{\dagger}(t)-\mathbf{W}_{Q G}(t)$, under the same conditions as in Theorem 6.1, but with the weaker smoothness $\sigma-\alpha>5$ we have the estimate, for both inviscid and viscous cases:

$$
\begin{aligned}
\left\|\Pi^{Q G} \mathbf{U}^{\dagger}(t)-\mathbf{W}_{Q G}(t)\right\|_{\alpha} & \leq C R o_{a} a_{3} / \mu_{2}, \text { for } f>N \\
& \leq C a_{3}^{2} /\left(N \mu_{2}\right), \text { for } f<N
\end{aligned}
$$


The same estimates hold for $\left\|\overline{\overline{\mathbf{U}}}^{\dagger}(t)-\overline{\overline{\mathbf{W}}}_{Q G}(t)-\overline{\overline{\mathbf{E}(-t) \mathbf{w}}}_{A G}(t)\right\|_{\alpha}$, but with $\sigma-\alpha>4$. Here $\overline{\overline{\mathbf{U}}}^{\dagger}$ designates the vertical averaging.

\section{Remark 6.1:}

For the full error, the above requires smoothness of initial data in $H_{9}$. For the convergence of the QG component, only $H_{7}$. This is a substantial improvement over the $H_{37}$ in BMN (1996a) and the $H_{18}$ in BMN (1996b). The convergence under the $H_{6}$ smoothness for the vertically averaged fields is rather remarkable, as it involves both the QG component and the AG component. It clearly shows that the Dynamical TaylorProudman theorem established in BMN $(1996 \mathrm{a}, \mathrm{b})$ has a modified version for the $B u=O(1)$ case, coupling $\mathrm{QG}$ and $\mathrm{AG}$ component.

In Theorem 6.1, the measures $\mu_{2}$ and $\mu_{3}$ are equal to the measures of the excluded sets of $a_{2}^{2}$ and $B u$; they reflect estimates of the measure of almost resonant $a_{2}$ or $B u$. In fact, such estimates imply the "Arnold Tongues" of Figure 2:

\section{Corollary 6.1:}

Let $N>f$ and $1 / B u, \theta_{2}=1 / a_{2}^{2}$ be the resonance parameters, with $1 / B_{M} \leq 1 / B u \leq 1 / B_{m}$, and $B_{m}=O(1)$. Then

$$
\mu_{3}\left(1 / B u, \theta_{2}, \theta_{3}\right)=\tilde{C} a_{3}^{4}
$$

where $\tilde{C}$ is an absolute constant independent from $\theta_{2}, \theta_{3}$ and $\tilde{C}=O(1)$. The probabilistic (normalized) measure of near 3 -wave resonances in $1 / B u$ on the interval $\left[0,1 / B_{m}\right]$ is $\tilde{C} B_{m} a_{3}^{2}$, and the factor $a_{3}^{4} / \mu_{3}$ within the error estimate in Theorem 6.1 reduces to $\tilde{C}$.

\section{Corollary 6.2:}

Let $N<f$ and $B u, \theta_{2}=1 / a_{2}^{2}$ be the resonance parameters, with $0 \leq B u \leq a_{3}^{2}$. Then

$$
\mu_{3}\left(B u, \theta_{2}, \theta_{3}\right)=C^{*} a_{3}^{3}
$$

where $C^{*}$ is an absolute constant independent from $\theta_{2}, \theta_{3}, C^{*}=O(1)$. The probabilistic (normalized) measure of near 3 -wave resonances in $B u$ on the interval $\left[0, a_{3}^{2}\right]$ is $C^{*} a_{3}^{3}$, and the factor $a_{3}^{3} / \mu_{3}$ within the error estimate in Theorem 6.1 reduces to $C^{*}$.

\section{Remark 6.2:}

For $N>f, 1 / B u=\left(f / N a_{3}\right)^{2}$ is the natural resonance parameter; $B u \rightarrow+\infty, N \rightarrow+\infty$ in the strongly stratified limit. For $N<f ; B u \rightarrow 0$ as $f \rightarrow+\infty$ and $B u=\left(N a_{3} / f\right)^{2}$ is automatically smaller than $a_{3}^{2}$.

Corollary 6.1 proves that the probability (normalized measure) of near 3 -wave resonances at $B u=$ $O(1)$ is very small for the atmosphere on synoptic and mesoscales; $H \approx 10 \mathrm{~km}$, and even for borderline small/mesoscales $L \approx 50 \mathrm{~km}, a_{3}^{2} \approx 4 \times 10^{-2}$. The 3-wave almost resonant sets for such domains are very sparse, quantitatively confirming Bartello's (1995) picture. 
Using approximations of solutions of 3D rotating Euler-Boussinesq equations by solutions of the QG and AG equations, we have proven global regularity of solutions of 3D Euler-Boussinesq equations with arbitrary large initial data on arbitrary long time intervals. The global existence theorems in $H_{3}$ for QG equations obtained by Bourgeois and Beale, as well as the conservation of energy on the restricted uncoupled families of 4 rays for $\mathbf{W}_{A G}$ ensure global existence for $\mathbf{W}_{Q G}+\mathbf{W}_{A G}$. Contrary to Bourgeois and Beale (1994), we do not require any "prepared" initial data:

\section{Theorem 6.3:}

Let $1 / B u \notin \Theta_{B}^{\mu_{3}}\left(\theta_{2}, \theta_{3}\right), \theta_{2} \notin \Theta_{2}^{\mu_{2}}, B_{m} \leq B u \leq B_{M}$. Let $N>f, \nu_{1}=\nu_{2}=0$. Let $\sigma>9$, and $M_{\sigma}>0$, $T^{*}>0$ be arbitrarily large. Then there exists $N^{*}=N^{*}\left(M_{\sigma}, T^{*}, \mu_{3}, \mu_{2}\right)$ such that for $\left\|\mathbf{U}^{\dagger}(0)\right\|_{\sigma} \leq M_{\sigma}$ and $N \geq N^{*}$, there exists a unique regular solution $\mathbf{U}^{\dagger}(t)$ of the 3D rotating Euler-Boussinesq equations which belongs to $H_{\sigma}$ for $0 \leq t \leq T^{*}$. For $M_{\sigma}$ fixed, $T^{*} \rightarrow+\infty$ as $N^{*} \rightarrow+\infty$ with explicit uniform dependence of $T^{*}$ on $M_{\sigma}, \mu_{3}, \mu_{2}, N^{*}$. Simultaneously, we can take arbitrary large (but bounded) set of initial data: $M_{\sigma} \rightarrow+\infty$ if $N^{*} \rightarrow+\infty$, for fixed $T^{*}$.

If we do not require explicit uniform estimates of $T^{*}$ (equivalently $N^{*}$ ) in terms of the geometric parameters $a_{2}, a_{3}$ and the measures $\mu_{2}, \mu_{3}$, we can obtain the following "Poor Man's" long-time existence theorem with weaker smoothness assumptions, valid for non-resonant $B u$, but holding for all $\theta_{2}$ and $a_{2}$ and all $\theta_{3}$ and $a_{3}$ (no constraints on 2 -wave resonances):

\section{Theorem 6.4:}

Let $N>f, \nu_{1}=\nu_{2}=0, B_{n} \leq B u \leq B_{M}, 1 / B u \notin \Theta_{B}^{*}\left(\theta_{2}, \theta_{3}\right)$. Let $M_{4}>0, T^{\prime}>0$ be arbitrary large. Then there exists $N_{1}$ such that if $\left\|\mathbf{U}^{\dagger}(0)\right\|_{4} \leq M_{4}$ and $N \geq N_{1}$, there exists a unique regular solution $\mathbf{U}^{\dagger}(t)$, $0 \leq t \leq T^{\prime}$ of the $3 \mathrm{D}$ rotating Euler-Boussinesq equations, which belongs to $H_{4}$ as $0 \leq t \leq T^{\prime}$. For $M_{4}$ fixed, $T^{\prime} \rightarrow+\infty$ with $N_{1} \rightarrow+\infty$. Simultaneously we can take arbitrary large (but bounded) sets of initial data: $M_{4} \rightarrow+\infty$ if $N_{1} \rightarrow+\infty$, for fixed $T^{\prime \prime}$. The above holds for all $a_{2}, a_{3}, 0<a_{2}, a_{3} \leq 1$.

Finally, using techniques from BMN (1996a, b, d) and the fact that the effective spectral eddy viscosities lie between $\nu_{1}$ and $\nu_{2}$ (see Section 3), we obtain the regularity for all times for the 3D rotating BoussinesqNavier Stokes equations (the so called "primitive" equations, not to be confused with equations associated to hydrostatic pressure hypothesis). This theorem describes the situation when $N$ is fixed, large enough and $B_{m} \leq B u \leq B_{M}$. The Navier-Stokes equations are forced by a force $\mathbf{F}(t)$ smooth enough. The situation is that of non-smooth and arbitrary large initial data in $H_{0}$. Then weak Leray solutions exist with maybe a blow-up in $H_{1}$.

\section{Theorem 6.5:}

Let $\nu_{1}>0, \nu_{2}>0, N>f, B_{m} \leq B u \leq B_{M}, 1 / B u \notin \Theta_{B}^{*}\left(\theta_{2}, \theta_{3}\right)$. Let $\|\mathbf{F}\|_{4}+\left\|\partial_{t} \mathbf{F}\right\|_{1} \leq M_{04}$ for all $t \geq 0,\left\|\mathbf{U}^{\dagger}(0)\right\|_{0} \leq M_{0}$. Let $\hat{T}=M_{0}^{2} / \nu_{m}$, where $\nu_{n}=\min \left(\nu_{1}, \nu_{2}\right) ; \hat{T}$ depends only on $\left\|\mathbf{U}^{\dagger}(0)\right\|_{0}, \nu_{1}, \nu_{2}$. 
Let $N^{\prime}$ be a number which depends only on $M_{04}, \nu_{1}, \nu_{2}, a_{2}, a_{3}$. Then for every fixed $N \geq N^{\prime}$ and for any weak solution $\mathbf{U}^{\dagger}(t)$ of the $3 \mathrm{D}$ rotating Boussinesq-Navier-Stokes equations which is defined on $[0, \hat{T}]$ and

satisfies the classical energy inequality on $[0, \hat{T}]$, the following proposition is true: $\mathbf{U}^{\dagger}(t)$ can be extended to $0<t<+\infty$ and it is regular for $\hat{T} \leq t<+\infty$; it belongs to $H_{4}$ and $\left\|\mathbf{U}^{\dagger}(t)\right\|_{4} \leq C_{1}\left(M_{04}, \nu_{1}, \nu_{2}\right)$ for every $t \geq \hat{T}$. It $\mathbf{F}$ is time-independent there exists a global attractor of the "primitive" equations of geophysics, which is bounded in $H_{4}$, has a finite fractal dimension, and every weak solution is attracted to the global attractor as $t \rightarrow+\infty$.

\section{Remark 6.3:}

No "preparation" of initial conditions is needed, contrary to the special restricted results of Chemin (1995). This theorem holds for all $a_{2}, 0<a_{2} \leq 1$. This theorem resolves problems of existence of attractors of for the "primitive" equations of geophysics raised by Lions, Temam and Wang (1994). These authors are able to give only conditional theorems assuming a priori regularity in $3 \mathrm{D}$. We demonstrate the regularizing effects of mixed rotation/stratification in the $B u=O(1)$ regimes.

\section{Concluding remarks}

In this paper, we have treated the problem of strongly stratified limit of rotating Boussinesq equations via two complementary approaches. First, we have illustrated the procedure for obtaining the dynamical decoupling of the vortical and inertial-gravity wave components of the total flow field. The 'split' of the energy transfer of the vortical and the wave components is established. As a result, analysis of 3D Boussinesq system has been reduced to several simple, well defined steps. We give explicit phase formulas for the Doppler phase shift induced by the interaction between the waves and the mean flow. In the regime of strong stratification and weak rotation we proposed regularized reduced equations with control of vertical shearing for all times. The regularizing effect of weak rotation principally differs from previously known regularizations which are based on vertical viscosity. Second, we have utilized the fact that the time scales of vortical and inertial-gravity wave components are disparate. Several useful results can be deduced without going through the steps outlined above. For ageostrophic dynamics, we demonstrate gradual unfreezing of energy cascades as $B u$ varies from zero to infinity. The energy spectrum and the anisotropic spectral eddy viscosity can be derived with an explicit dependence on the anisotropic rotation/stratification time scale. This time scale, in turn, depends on the aspect ratio parameter (ratio of the vertical and the horizontal length scale). Our analysis provides a potential context for investigations of the Garrett-Munk spectrum induced by the interaction between inertio-gravity waves and turbulence (Garrett and Munk, 1979). 


\section{Acknowledgements}

The authors wish to thank for their support the AFOSR (grant F49620-96-0-0165) and the ASU Centers for Environmental Fluid Dynamics and Systems Science and Engineering. The hospitality of the Newton Institute (Cambridge) under the special program "Mathematics of Atmosphere and Ocean Dynamics" is gratefully acknowledged. We would like to thank Professors C.W. Bardos, P. Bartello, M.J.P. Cullen, M. Farge, J.R. Herring, J.C.R. Hunt, J.C. McWilliams, P.S. Marcus, H.K. Moffatt, J. Tribbia, G.K. Vallis, T. Warn and V. Zeitlin for very illuminating discussions.

\section{A Appendix: Dependence of the coefficients $Q_{k m n}^{i_{1} i_{2} i_{3}}$ on $\mu=f / N$}

The coefficients $Q_{k m n}^{i_{1} i_{2} i_{3}}(\mu)$ given by Eqs. (2.25)-(2.28) can be expanded in powers of $\mu=f / N$ as follows

$$
\begin{aligned}
& Q_{k m n}^{012}(\mu)=-2 \mu \frac{\xi_{m} \xi_{n}\left(k^{\prime} \wedge m^{\prime}\right)^{2}}{\phi_{n}\left|k^{\prime}\left\|m^{\prime}\right\| n^{\prime}\right|}+O\left(\mu^{3}\right) \\
& Q_{m k n}^{102}(\mu)=-2 \mu \frac{\left(m_{3} k^{\prime} \cdot m^{\prime}-k_{3}\left|m^{\prime}\right|^{2}\right)\left(n_{3} n^{\prime} \cdot k^{\prime}-k_{3}\left|n^{\prime}\right|^{2}\right)}{\left|k^{\prime}\right|\left|n^{\prime}\right|\left|m \| m^{\prime}\right|\left|n^{\prime}\right|}+O\left(\mu^{3}\right) \\
& Q_{k m n}^{021}(\mu)=2 \mu \frac{\xi_{m} \xi_{n}\left(k^{\prime} \wedge m^{\prime}\right)^{2}}{\phi_{m}\left|k^{\prime}\right|\left|m^{\prime}\right|\left|n^{\prime}\right|}+O\left(\mu^{3}\right), Q_{m k n}^{201}(\mu)=2 \mu \frac{\xi_{m} \xi_{n}\left(k^{\prime} \wedge m^{\prime}\right)^{2}}{\phi_{m}\left|k^{\prime}\right|\left|m^{\prime}\right|\left|n^{\prime}\right|}+O\left(\mu^{3}\right) \\
& Q_{k m n}^{011}(\mu)=Q_{k m n}^{011}(0)-\frac{1}{2} \mu^{2} \frac{k_{3}^{2}\left(k^{\prime} \wedge m^{\prime}\right)\left(n_{3} m_{3} n^{\prime} \cdot m^{\prime}+\left|n^{\prime}\right|^{2}\left|m^{\prime}\right|^{2}\right)}{\left|k^{\prime}\right|^{3}\left|m^{\prime}\right|\left|n^{\prime}\right||m||n|}+O\left(\mu^{4}\right), \\
& Q_{m k n}^{101}(\mu)=Q_{m k n}^{101}(0)-\frac{1}{2} \mu^{2} \frac{k_{3}^{2} \xi_{n}\left(k^{\prime} \wedge m^{\prime}\right)\left(m_{3} k^{\prime} \cdot m^{\prime}-k_{3}\left|m^{\prime}\right|^{2}\right)}{\left|k^{\prime}\right|^{3}|m|\left|m^{\prime}\right|\left|n^{\prime}\right|}+O\left(\mu^{4}\right) \\
& Q_{k m n}^{022}(\mu)=Q_{k m n}^{022}(0)-\frac{1}{2} \mu^{2} \frac{k^{\prime} \wedge m^{\prime}}{\left|k^{\prime}\right|}\left(\frac{k_{3}^{2}}{\left|k^{\prime}\right|^{2}}+\frac{m_{3}^{2}}{\left|m^{\prime}\right|^{2}}+\frac{n_{3}^{2}}{\left|n^{\prime}\right|^{2}}\right)+\mu^{2} \frac{\left(k^{\prime} \wedge m^{\prime}\right) m_{3} n_{3} m^{\prime} \cdot n^{\prime}}{\left|k^{\prime}\right|\left|m^{\prime}\right|\left|n^{\prime}\right|\left|m^{\prime}\right|\left|n^{\prime}\right|}+O\left(\mu^{4}\right), \\
& Q_{m k n}^{202}(\mu)=Q_{m k n}^{202}(0)+\mu^{2} \frac{m_{3}\left(k^{\prime} \wedge m^{\prime}\right)\left(k_{3}\left|n^{\prime}\right|^{2}-n_{3} n^{\prime} \cdot k^{\prime}\right)}{\left|k^{\prime}\right|\left|m^{\prime}\right|\left|n^{\prime}\right|\left|m^{\prime}\right|\left|n^{\prime}\right|}+O\left(\mu^{4}\right), \text { where } \\
& Q_{k m n}^{011}(0)=\frac{k^{\prime} \wedge m^{\prime}}{\left|k^{\prime}\right|\left|m^{\prime}\right|\left|n^{\prime}\right||m||n|}\left(n_{3} m_{3} n^{\prime} \cdot m^{\prime}+\left.\left|m^{\prime 2}\right| n^{\prime}\right|^{2}\right), \quad Q_{k m n}^{022}(0)=\frac{1}{\left|k^{\prime}\right|}\left(k^{\prime} \wedge m^{\prime}\right), \\
& Q_{m k n}^{101}(0)=\frac{\xi_{n}\left(k^{\prime} \wedge m^{\prime}\right)\left(m_{3} k^{\prime} \cdot m^{\prime}-k_{3}\left|m^{\prime}\right|^{2}\right)}{\left|k^{\prime}\right|\left|m^{\prime}\right|\left|n^{\prime}\right||m|}, Q_{m k n}^{202}(0)=0 \\
& Q_{k m n}^{012}(0)=Q_{k m n}^{021}(0)=Q_{m k n}^{102}(0)=Q_{m k n}^{201}(0)=0 .
\end{aligned}
$$




\section{References}

Avrin, J., Babin, A., Mahalov, A., and Nicolaenko, B. (1996), Asymptotics for solutions of Boussinesq equations, submitted to J. Asympt. Anal.

Babin, A., Mahalov, A., and Nicolaenko, B. (1995), Long-time averaged Euler and Navier-Stokes equations for rotating fluids, In "Structure and Dynamics of Nonlinear Waves in Fluids", 1994 IUTAM Conference, K. Kirchgässner and A. Mielke (eds), World Scientific, 145-157.

Babin, A., Mahalov A., and Nicolaenko, B. (1996a), Global splitting, integrability and regularity of 3D Euler and Navier-Stokes equations for uniformly rotating fluids, Europ. J. of Mech., B/Fluids, 15, No. 3, 291-300. Babin, A., Mahalov, A., and Nicolaenko, B. (1996b), Resonances and regularity for Boussinesq equations, Russian J. Math. Phys., 4, No. 4, 417-428.

Babin, A., Mahalov, A., and Nicolaenko, B. (1996c), Regularity and integrability of rotating shallow-water equations, Proc. Acad. Sci. Paris, in press.

Babin, A., Mahalov, A., and Nicolaenko, B. (1996d), Global regularity and integrability of 3D Euler and Navier-Stokes equations for uniformly rotating fluids, Ann. Ecole Norm. Super., in press.

Babin, A., Mahalov, A., and Nicolaenko, B. (1996e), Global splitting and regularity of rotating shallow-water equations, J. Asympt. Anal., in press.

Bartello, P. (1995), Geostrophic adjustment and inverse cascades in rotating stratified turbulence, J. Atm. Sci., 52, No. 24, 4410-4428.

Bartello, P., and Holloway, G. (1991), Passive scalar transport in $\beta$-plane turbulence, J. Fluid Mech., 223, $521-536$.

Bartello, P., Métais, O., and Lesieur, M. (1994), Coherent structures in rotating three-dimensional turbulence, J. Fluid Mech, , 273, 1-29.

Bartello, P., Métais, O., and Lesieur, M. (1996), Geostrophic versus wave eddy viscosities in atmospheric models, J. Atm. Sci., 53, No. 4, 564-571.

Bourgeois, A.J., and Beale, J.T. (1994), Validity of the quasigeostrophic model for large-scale flow in the atmosphere and the ocean, SIAM J. Math. Anal., 25, No. 4, 1023-1068.

Carnevale, G. and Martin, P. (1982), Field theoretical techniques in statistical fluid dynamics: with applications to nonlinear wave dynamics, Geophys. Astrophys. Fluid Dyn., 20, 131-164.

Chemin, J.-Y. (1995), A propos d'un probleme de pénalisation de type antisymétrique, Proc. Paris Acad. Sci., 321, 861-864.

Doering, C., and Constantin, P. (1996), Variational bounds on energy dissipation in incompressible flows. Convection. Phys. Rev. E, 53, 5957-5981. 
Embid, P.F., and Majda, A.J. (1996), Averaging over fast gravity waves for geophysical flows with arbitrary potential vorticity, Comm. Partial Diff. Eqs., 21, 619-658.

Farge, M. (1988), Effects of inertiogravity waves and rotation on two-dimensional turbulence, Current Trends in Turbulence Research, Vol. 112 of Progress in Astronautics and Aeronautics, AIAA, 355-373.

Farge, M., and Sadourny, R. (1989), Wave-vortex dynamics in rotating shallow layer, J. Fluid Mech., 206, 433-462.

Fernando, H.J.S., and Hunt, J.C.R. (1996), Some aspects of turbulence and mixing in stably stratified layers, Dyn. of Atm. and Oceans, 23, 35.

Garrett, C., and Munk, W. (1979), Internal waves in the ocean, Ann. Rev. Fluid Mech., 11, 339-369.

Gent, P.R., and McWilliams, J.C. (1983a), Consistent balanced models in bounded and periodic domains, Dyn. Atm. and Oceans, 7, 67-93.

Gent, P.R., and McWilliams, J.C. (1983b), Regimes of validity for balanced models, Dyn. Atm. and Oceans, 7, 167-183.

Godeferd, F.S., and Cambon, C. (1994), Detailed investigation of energy transfer in homogeneous stratified turbulence, Phys. of Fluids, 6, No. 6, 2084-2100.

Herring, J.R., and McWilliams, J.C. (1985), Comparison of direct numerical simulations of two-dimensional turbulence with two-point closure. The effects of intermittency. J. Fluid Mech., 153, 229-242.

Herring, J.R., and Métais, O. (1989), Numerical experiments in forced stably stratified turbulence, J. Fluid Mech., 202, 97 .

Holton, J.R. (1992), An Introduction to Dynamic Meteorology, Academic Press.

Hopfinger, E.J. (1989), Turbulence and vortices in rotating fluids, Theor. and Appl. Mech., 125, 117-138.

Howard, L. (1972), Bounds on flow quantities, Ann. Rev. Fluid Mech., 4, 473-494.

Kimura, Y., and Herring, J.R. (1996), Diffusion in stably stratified turbulence, J. Fluid Mech. in press.

Kraichnan, R.H. (1965), Inertial range spectrum of hydromagnetic turbulence, Phys. of Fluids 8, 1385.

Legras, B. (1980), Turbulent phase shift of Rossby waves, Geophys. Astrophys. Fluid Dyn., 15, 253-281.

Lelong, M.-P., and Riley, J. (1991), Internal wave-vortical mode interactions in strongly stratified flows, $J$. Fluid Mech., 232, 1-19.

Lesieur, M. (1987), Turbulence in Fluids, Martinus Nijhoff Publishers.

Lilly, D.K. (1983), Stratified turbulence and the mesoscale variability of the atmosphere, J. Atm. Sc., 40, 749 .

Mahalov, A. (1993), The instability of rotating fluid columns subjected to a weak external Coriolis force, Phys. Fluids A, 5, No. 4, 891-900. 
Mahalov, A. and Marcus, P.S. (1995), Long-time averaged rotating shallow-water equations, Proc. of the First Asian Computational Fluid Dynamics Conference, W.H. Hui, Y.-K. Kwok and J.R. Chasnov, (eds.) vol. 3, 1227-1230, Hong Kong University of Science and Technology.

Mahalov, A., and Zhou, Y. (1996), Analytical and phenomenological studies of rotating turbulence, Phys. of Fluids, 8, No. 8, 2138-2152.

McWilliams, J.C. (1985), A note on a uniformly valid model spanning the regimes of geostrophic and isotropic stratified turbulence: balanced turbulence, J. Atm. Sci., 42, 1773-1774.

McWilliams, J.C., and Gent, P.R. (1980), Intermediate models of planetary circulations in the atmosphere and ocean, J. Atm. Sci., 37, 1657-1678.

McWilliams J.C., Weiss, J.B., and Yavneh, I. (1994), Anisotropy and coherent vortex structures in planetary turbulence, Science, 264, 410-413.

Métais, O., and Herring, J.R. (1989), Numerical experiments of freely evolving turbulence in stably stratified fluids, J. Fluid Mech., 202, 117.

Métais, O., Bartello, P., Garnier, E., Riley, J.J., and Lesieur, M. (1996), Inverse cascades in stably stratified rotating turbulence, Dyn. of Atm. and Oceans, 23, 193-203.

Müller, P., Holloway, G., Henyey, F., and Pomphrey, N. (1986), Nonlinear interactions among internal gravity waves, Reviews of Geophysics, 24, No. 3, 493-536.

Pedlosky, J. (1987), Geophysical Fluid Dynamics, 2nd edition, Springer-Verlag.

Pushkarev, A.N., and Zakharov, V.E. (1996), Turbulence in capillary waves, Phys. Rev. Lett., 76, 3320-3323. Reynolds, W.C. and Kassinos, S.C. (1996), One-point modelling of rapidly deformed homogeneous turbulence, Proc. R. Soc. London Ser. A, 451, 87.

Riley, J.J., Metcalfe, R.W., and Weisman, M.A. (1981), Direct numerical simulations of homogeneous turbulence in density-stratified fluids, Proc. AIP Conf. Nonl. Prop. of Internal Waves, B.J. West (ed.), 79.

Ristorcelli, J.R., Lumley, J.L., and Abid, R. (1995), A rapid-pressure covariance representation consistent with the Taylor-Proudman theorem materially frame indifferent in the two-dimensional limit, J. Fluid Mech., 292, 111-152.

Speziale, C.G. (1989), Turbulence modeling in noninertial frames of reference, Theor. and Comp. Fluid Dyn., 1, 3-19.

Warn, T. (1986), Statistical mechanical equilibria of the shallow-water equations, Tellus, 38A, 1-11.

Zakharov, V.E., Falkovich, G., and Lvov, V.S. (1992), Kolmogorov Spectra of Turbulence, Springer-Verlag. Zhou, Y. (1995), A phenomenological treatment of rotating turbulence, Phys. Fluids, 7, 2092. 


\section{REPORT DOCUMENTATION PAGE}

Form Approved

OMB No. $0704-0188$

Public reporting burden for this collection of information is estimated to average 1 hour per response, including the time for reviewing instructions, searching existing data sources, gathering and maintaining the data needed, and completing and reviewing the collection of information. Send comments regarding this burden estimate or any other aspect of this collection of information, including suggestions for reducing this burden, to Washington Headquarters Services, Directorate for Information Operations and Reports, 1215 Jefferson Davis Highway, Suite 1204, Arlington, VA 22202-4302, and to the Office of Management and Budget, Paperwork Reduction Project (0704-0188), Washington, DC 20503.

\begin{tabular}{|c|c|c|}
\hline 1. AGENCY USE ONLY(Leave blank) & $\begin{array}{l}\text { 2. REPORT DATE } \\
\text { March } 1997\end{array}$ & $\begin{array}{l}\text { 3. REPORT TYPE AND DATES COVERED } \\
\text { Contractor Report }\end{array}$ \\
\hline
\end{tabular}

4. TITLE AND SUBTITLE 5. FUNDING NUMBERS

On the asymptotic regimes and the strongly stratified limit of rotating

Boussinesq equations

C NAS1-19480

WU 505-90-52-01

6. AUTHOR(S)

A. Babin, A. Mahalov,

B. Nicolaenko, and Y. Zhou

7. PERFORMING ORGANIZATION NAME(S) AND ADDRESS(ES)

Institute for Computer Applications in Science and Engineering

Mail Stop 403, NASA Langley Research Center

Hampton, VA 23681-0001

8. PERFORMING ORGANIZATION REPORT NUMBER

ICASE Report No. 97-18

9. SPONSORING/MONITORING AGENCY NAME(S) AND ADDRESS(ES)

National Aeronautics and Space Administration

Langley Research Center

Hampton, VA. 23681-0001

10. SPONSORING/MONITORING AGENCY REPORT NUMBER

NASA CR-201672

ICASE Report No. 97-18

\section{SUPPLEMENTARY NOTES}

Langley Technical Monitor: Dennis M. Bushnell

Final Report

Submitted to Theoretical and Computational Fluid Dynamics.

12a. DISTRIBUTION/AVAILABILITY STATEMENT

12b. DISTRIBUTION CODE

Unclassified-Unlimited

Subject Category 34

13. ABSTRACT (Maximum 200 words)

Asymptotic regimes of geophysical dynamics are described for different Burger number limits. Rotating Boussinesq equations are analyzed in the asymptotic limit of strong stratification in the Burger number of order one situation as well as in the asymptotic regime of strong stratification and weak rotation. It is shown that in both regimes horizontally averaged buoyancy variable is an adiabatic invariant for the full Boussinesq system. Spectral phase shift corrections to the buoyancy time scale associated with vertical shearing of this invariant are deduced. Statistical dephasing effects induced by turbulent processes on inertial-gravity waves are evidenced. 'The 'split' of the energy transfer of the vortical and the wave components is established in the Craya-Herring cyclic basis. As the Burger number increases from zero to infinity, we demonstrate gradual unfreezing of energy cascades for ageostrophic dynamics. The energy spectrum and the anisotropic spectral eddy viscosity are deduced with an explicit dependence on the anisotropic rotation/stratification time scale which depends on the vertical aspect ratio parameter. Intermediate asymptotic regime corresponding to strong stratification and weak rotation is analyzed where the effects of weak rotation are accounted for by an asymptotic expansion with full control (saturation) of vertical shearing. The regularizing effect of weak rotation differs from regularizations based on vertical viscosity. Two scalar prognostic equations for ageostrophic components (divergent velocity potential and geostrophic departure) are obtained.

\begin{tabular}{|c|c|c|c|}
\hline \multicolumn{3}{|c|}{$\begin{array}{l}\text { 14. SUBJECT TERMS } \\
\text { turbulence; stratification; rotation }\end{array}$} & $\begin{array}{c}\text { 15. NUMBER OF PAGES } \\
43\end{array}$ \\
\hline $\begin{array}{l}\text { 17. SECURITY CLASSIFICATION } \\
\text { OF REPORT } \\
\text { Unclassified }\end{array}$ & $\begin{array}{l}\text { 18. SECURITY CLASSIFICATION } \\
\text { OF THIS PAGE } \\
\text { Unclassified }\end{array}$ & $\begin{array}{l}\text { 19. SECURITY CLASSIFICATION } \\
\text { OF ABSTRACT }\end{array}$ & $\begin{array}{l}\text { 20. LIMITATION } \\
\text { OF ABSTRACT }\end{array}$ \\
\hline
\end{tabular}

